\title{
The Solar Mass Ejection Imager and Its Heliospheric Imaging Legacy
}

\author{
T.A. Howard • M.M. Bisi • A. Buffington · J.M. Clover · M.P. Cooke · C.J. Eyles • \\ P.P. Hick • P.E. Holladay • B.V. Jackson · J.C. Johnston • S.W. Kahler • T.A. Kuchar • \\ D.R. Mizuno • A.J. Penny • S.D. Price · R.R. Radick • G.M. Simnett • S.J. Tappin • \\ N.R. Waltham • D.F. Webb
}

Received: 19 December 2012 / Accepted: 16 April 2013 / Published online: 13 June 2013

(C) The Author(s) 2013. This article is published with open access at Springerlink.com

\begin{abstract}
The Solar Mass Ejection Imager (SMEI) was the first of a new class of heliospheric and astronomical white-light imager. A heliospheric imager operates in a fashion similar to coronagraphs, in that it observes solar photospheric white light that has been Thomson scattered by free electrons in the solar wind plasma. Compared with traditional
\end{abstract}

S.D. Price is deceased.

T.A. Howard $(\bowtie)$

Southwest Research Institute, 1050 Walnut Street, Suite 300, Boulder, CO 80302, USA

e-mail: howard@boulder.swri.edu

M.M. Bisi

Institute of Mathematical and Physical Sciences, Aberystwyth University, Ceredigion, SY23 3BZ, UK

A. Buffington - J.M. Clover · P.P. Hick · B.V. Jackson

Center for Astrophysics and Space Sciences, University of California, San Diego, CA 92093-0424, USA

M.P. Cooke · C.J. Eyles · G.M. Simnett

School of Physics and Astronomy, University of Birmingham, Edgbaston, B15 2TT, UK

C.J. Eyles

Laboratorio de Procesado de Imagenes, Universidad de Valencia, 46071, Valencia, Spain

C.J. Eyles · N.R. Waltham

RAL Space, Rutherford Appleton Laboratory, Chilton, Didcot, Oxfordshire, OX11 0QX, UK

P.E. Holladay

Rust Street, Hamilton, MA 01982, USA

J.C. Johnston

44th Street, Newbury, MA 01951, USA

S.W. Kahler

Air Force Research Laboratory, Kirtland AF Base, Albuquerque, NM 87117, USA

T.A. Kuchar · D.R. Mizuno - S.D. Price · D.F. Webb

Institute for Scientific Research, Boston College, Newton, MA 02459, USA 
coronagraphs, this imager differs in that it observes at much larger angles from the Sun. This in turn requires a much higher sensitivity and wider dynamic range for the measured intensity. SMEI was launched on the Coriolis spacecraft in January 2003 and was deactivated in September 2011, thus operating almost continuously for nearly nine years. Its primary objective was the observation of interplanetary transients, typically coronal mass ejections (CMEs), and tracking them continuously throughout the inner heliosphere. Towards this goal it was immediately effective, observing and tracking several CMEs in the first month of mission operations, with some 400 detections to follow. Along with this primary science objective, SMEI also contributed to many and varied scientific fields, including studies of corotating interaction regions (CIRs), the high-altitude aurora, zodiacal light, Gegenschein, comet tail disconnections and motions, and variable stars. It was also able to detect and track Earth-orbiting satellites and space debris. Along with its scientific advancements, SMEI also demonstrated a significantly improved accuracy of space weather prediction, thereby establishing the feasibility and usefulness of operational heliospheric imagers. In this paper we review the scientific and operational achievements of SMEI, discuss lessons learned, and present our view of potential next steps in future heliospheric imaging.

Keywords Instrumentation · Heliospheric imaging · Coronal mass ejections · Interplanetary medium $\cdot$ Solar-terrestrial relations $\cdot$ Corotating interaction regions · High-altitude aurora $\cdot$ Zodiacal light $\cdot$ Gegenschein $\cdot$ Comets · Variable stars · Space weather forecasting

\section{Introduction}

The last decade witnessed the emergence of a new class of visible white-light imager for heliospheric observations. A heliospheric imager is essentially a wide-angle version of a coronagraph, based on the same principle of observing sunlight photons which have been Thomson scattered by free electrons in the solar wind. Heliospheric imagers require a much higher sensitivity and larger dynamic range than coronagraphs, thus enabling them to observe across much greater angular spans in the sky, and to larger distances from the Sun. Heliospheric Imagers (HIs) are carried on board the twin spacecraft of the Solar Terrestrial Relations Observatory (STEREO) mission (Eyles et al. 2009), but the first such instrument was the Solar Mass Ejection Imager (SMEI). This instrument (Eyles et al. 2003; Jackson et al. 2004) was launched in 2003, de-activated in 2011, and operated almost continuously during its $8 \frac{1}{2}$-year lifetime. It observed almost the entire heliosphere from about $18^{\circ}$ outwards from the Sun ( $>0.35 \mathrm{AU}$ in the plane of the sky).

The heliospheric imaging legacy dates back to the Helios mission (Leinert et al. 1975), whose zodiacal light experiment observed interplanetary transients at large distances from the Sun in white light (Richter et al. 1982; Jackson and Leinert 1985; Jackson 1985a). Although over 20 years elapsed from the end of the Helios mission in 1982 to the launch of

A.J. Penny
SUPA, School of Physics and Astronomy, University of St. Andrews, St. Andrews, KY16 9SS, UK

R.R. Radick

Air Force Research Laboratory, National Solar Observatory, Sunspot, NM 88349, USA

S.J. Tappin

National Solar Observatory, Sunspot, NM 88349, USA 
SMEI in 2003, the SMEI concept dates back to the 1980s (Jackson 1985b). Jackson et al. (2004) provide a review of the evolution of the Helios design philosophy into that of SMEI. SMEI consisted of a multistage labyrinthine baffle (Jackson et al. 1989; Eyles et al. 2003; Buffington et al. 2003, and references therein) coupled to an imaging system (Eyles et al. 2003 ) with a $60^{\circ} \times 3^{\circ}$ useful photometric field of view.

The primary objective of SMEI was the observation and tracking of interplanetary transients, specifically coronal mass ejections (CMEs), as they move through the heliosphere. Towards this objective it was immediately effective, with several CMEs tracked during the first month of mission operations (e.g., Webb et al. 2006). A further objective was the determination of the time of arrival of CMEs at Earth. To this end SMEI was used to measure distances of heliospheric structures from Earth prior to their Earth arrival, or, better still, it enabled the deconvolution of the detailed distribution of heliospheric material along the line of sight (see Sect. 4.1.2). SMEI has also provided useful information to other scientific fields, including the high-altitude aurora, the zodiacal light, the Gegenschein, variable stars, corotating interaction regions, and comets. At the time of writing, the number of peer-reviewed papers involving SMEI totaled 113, with an additional 195 post-launch publications and conference proceedings and 32 press releases or public articles. These numbers continue to increase. In this review we discuss the SMEI mission and instrument, review its scientific and technical accomplishments, and conclude with a brief discussion of lessons learned and potential next steps for a future heliospheric imager.

\section{The SMEI Mission}

SMEI (Eyles et al. 2003; Jackson et al. 2004) was designed as a proof-of-concept instrument for forecasting the arrival of CMEs and for exploring their role in the occurrence of geomagnetic storms (Webb et al. 2002). It was launched on board the Air Force Space Test Program's Coriolis spacecraft from the Vandenberg Air Force Base on 6 January 2003 and was one of two instruments on Coriolis, the primary payload being the Naval Research Laboratory's WINDSAT microwave polarimetry experiment (Gaiser 2004). Coriolis was inserted into a dawn-dusk, Sun-synchronous polar orbit (orbital plane inclined at $98^{\circ}$ from the equator) at an altitude of $840 \mathrm{~km}$.

The SMEI instrument consisted of three cameras. Each camera was provided with a multi-stage labyrinthine baffle to reduce stray light (Buffington et al. 2003), together with a central Data Handling Unit (DHU) that controlled the readout of the cameras and provided the command and telemetry data interfaces with the spacecraft (Eyles et al. 2003). The cameras were mounted on the spacecraft in such a way that each observed a different part of the sky during their whole-orbit scan. Camera 1 observed the outermost field of view $\left(\sim 120^{\circ}-180^{\circ}\right.$ from the Sun), Camera 2 observed approximately $70^{\circ}-130^{\circ}$, while Camera 3 observed around $18^{\circ}-80^{\circ}$. Figure 1a shows a photograph of the Coriolis spacecraft with the three SMEI cameras indicated; Fig. 1b shows an illustration of SMEI in orbit on board Coriolis. Table 1 summarizes the key parameters of the SMEI instrument.

Early flight data confirmed that the stray-light rejection specification for SMEI was achieved over most of the field of view (FOV) (Buffington et al. 2005). As demonstrated by Buffington et al. (2006a, their Sect. 4 and Fig. 6), the SMEI photometric specification was met for the majority of the sky by Cameras 1 and 2. Performance for Camera 3, with its higher noise level, was relatively poor (see Sect. 3.2.1), and grew worse further throughout the mission. The quality of Camera 3, and to a lesser extent, Cameras 1 and 2, decreased mostly as a result of dark charge noise from hot pixel accumulation (see Sect. 3.2.1). 
Fig. 1 (a) The Coriolis spacecraft prior to launch with the three SMEI cameras indicated. (b) Schematic of SMEI in orbit. The SMEI cameras and their fields of view are depicted as approximately $30^{\circ}$ above the local horizon to avoid the light from the Earth and sunlight reflected from the rotating WINDSAT antenna. The baffles point backwards with respect to the orbital direction to reduce contamination

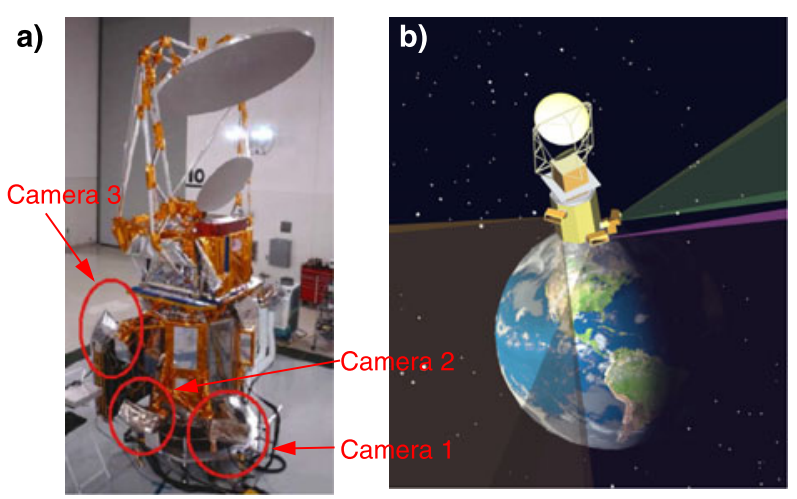

Table 1 SMEI mission and instrument specification. $B_{\odot}$ refers to the mean surface brightness of the Sun

\begin{tabular}{|c|c|}
\hline Parameter & Value \\
\hline Orbit & $\begin{array}{l}840 \mathrm{~km} \text { altitude, } 98^{\circ} \text { inclination, Sun-synchronous } \\
102 \text {-minute period }\end{array}$ \\
\hline Mission design & $\begin{array}{l}\text { Secondary payload on board Coriolis spacecraft } \\
\text { Nadir/velocity-vector stabilized }\end{array}$ \\
\hline Camera and optical design & $\begin{array}{l}\text { 2-element reflecting optics } \\
1.76 \mathrm{~cm}^{2} \text { aperture, } f / 1.2 \times f / 2.2\end{array}$ \\
\hline Field-of-view & $\begin{array}{l}3 \text { cameras each } 60^{\circ} \times 3^{\circ} \\
\text { Combined instantaneous FOV } \sim 160^{\circ} \times 3^{\circ}\end{array}$ \\
\hline Sky coverage (each orbit) & $\begin{array}{l}\text { All sky except for } \sim 18^{\circ} \text { radius exclusion region around Sunward } \\
\text { pole of orbit and smaller exclusion region in anti-Sun direction }\end{array}$ \\
\hline Differential photometric precision & $0.1 \%$, except for small regions near bright objects \\
\hline Angular resolution & $\sim 0.5^{\circ}$ width of point spread function (PSF) \\
\hline Band-pass & $450-950 \mathrm{~nm}(\mathrm{CCD}$ quantum efficiency $\geq 10 \%)$ \\
\hline Exposure cadence & 4 seconds \\
\hline Detector & $1280 \times 576$ pixel frame-store AIMO CCD \\
\hline Detector pixel scale & $0.05^{\circ} \times 0.05^{\circ}$ \\
\hline On-board pixel binning & $\begin{array}{l}\text { Cameras } 1 \text { and } 2: 4 \times 4 \mathrm{CCD} \text { pixel binning } \\
\text { Camera 3: } 2 \times 2 \mathrm{CCD} \text { pixel binning }\end{array}$ \\
\hline Detector temperature & $-30{ }^{\circ} \mathrm{C}($ design value $)$ \\
\hline Baffle concept / Stray light rejection & 2/3-stage rectangular knife-edge baffle system \\
\hline Average stray light & $<10^{-15} B_{\odot}$ \\
\hline Mass & $36 \mathrm{~kg}$ \\
\hline Average power & $22 \mathrm{~W}$ \\
\hline Average telemetry rate & 64 kbps (128 kbps in "Engineering Mode") \\
\hline
\end{tabular}

The protective doors of the three cameras were opened on 1 February 2003, the first day of usable observations was 5 February, and the first complete day of science observations was on 6 February. The first recorded CME occurred on 10 February, and a further 11 were documented by the end of that first month. The year 2003 was just after the peak of the Sun's activity cycle 23 . Figure 2 shows the duty cycle of science data throughout the 


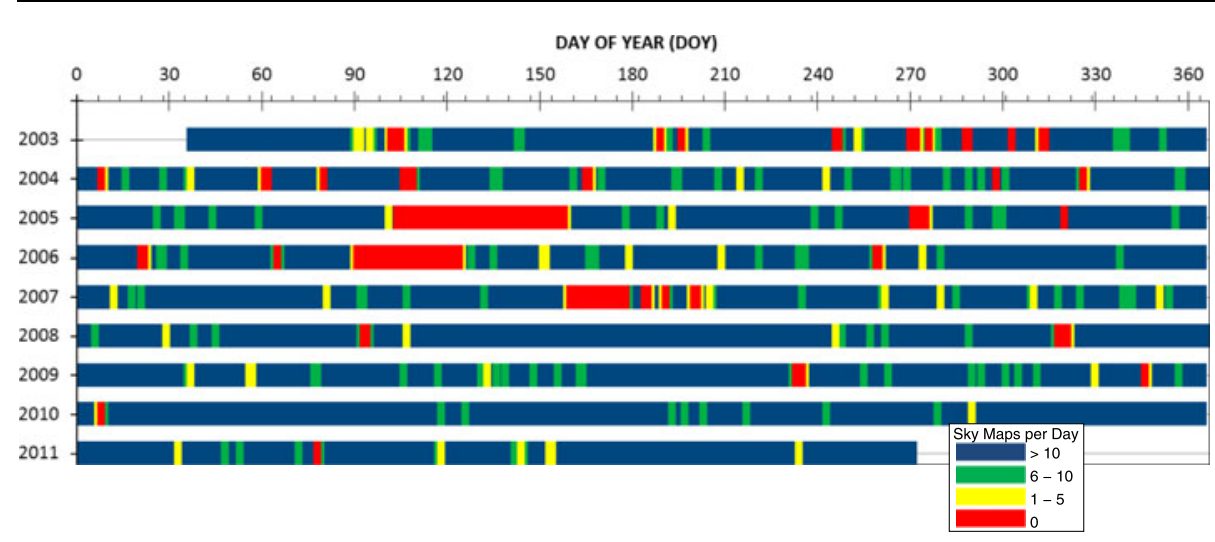

Fig. 2 Duty cycle for SMEI science data throughout the mission, from the first complete day of science observations (6 February 2003) to the shutdown on 28 September 2011. The color code indicates the number of usable sky maps each day, as labeled. As SMEI had a cadence of 102 minutes the majority of days had 14 maps, but some had 15 . The minor divisions on the $x$-axis (DOY) indicate weeks

SMEI mission. While small data gaps occurred throughout, three large gaps (large areas of red) occurred in 2005, 2006 and 2007. The 2005 and 2007 anomalies were caused by the Coriolis spacecraft transitioning to safe modes, in the former case as a result of the antenna bearings of WINDSAT locking up and in the latter case due to problems with the on-board star tracker. The anomalies were resolved in both cases but not without the loss of SMEI observations.

The 2006 gap was caused by the only significant hardware failure on SMEI during the mission. The "A"-side of the DHU failed during a passage of Coriolis through the South Atlantic Anomaly (SAA), requiring that control of the instrument be transferred to the "B"side DHU. Passages through regions of high particle background (SAA and polar regions) caused processor anomalies in the DHU typically every few weeks throughout the mission: each time required a subsequent processor re-boot for recovery. Prior to 2008 this was handled entirely manually, resulting in delays of some hours in restoring observations. However, in early 2008 new procedures were introduced, allowing the operations team to reboot SMEI without consulting the SMEI team for well-understood anomalies. This resulted in a much faster recovery following anomalies. Therefore Fig. 2 shows far fewer yellow and red bands after 2007 than in prior years.

SMEI was deactivated on 28 September 2011 after $8 \frac{1}{2}$ years operation. At the time of writing, Coriolis and WINDSAT remain operational. Presumably, SMEI could be reactivated if resources were available to support mission operations and the data processing efforts.

\section{SMEI Data Aspects}

The SMEI design enabled a nearly complete scan of the sky. Given the photometric and other specifications of Table 1 we believe this design is an excellent solution for a heliospheric imager in Earth orbit. Each of the three SMEI cameras imaged, with a 4-second exposure, a $3^{\circ} \times 60^{\circ}$ strip in the sky. The cameras were oriented such that their fields of view had $10^{\circ}$ overlaps in the $60^{\circ}$ direction, so the three cameras combined swept out approximately a $3^{\circ} \times 160^{\circ}$ great circle around the orbit axis, the pitch axis of the zenith-nadir pointed Coriolis spacecraft. The frame-store operation of the CCD cameras enabled exposures with minimal 


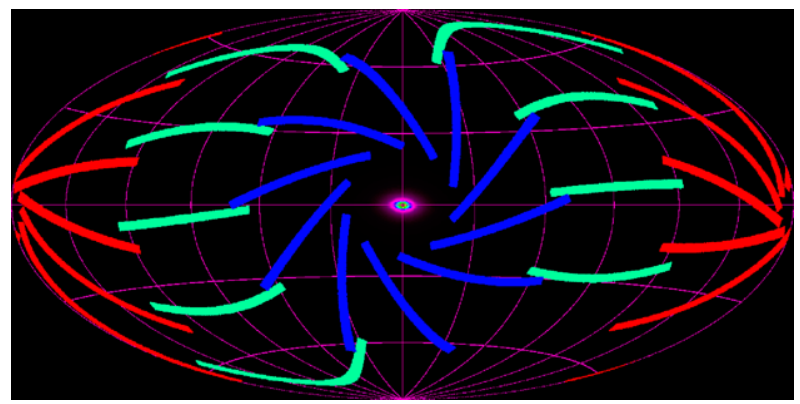

Fig. 3 An example of a Sun-centered Hammer-Aitoff (see, e.g., Leighly 1955) projection in ecliptic coordinates from 19 February 2003, showing how a selection of individual frames combine to produce a composite sky map (from Webb et al. 2006). The Sunward camera (Camera 3) frames are shown in blue, the next (Camera 2) in green, and the farthest from the Sun (Camera 1) in red. The gridlines (purple) mark $30^{\circ}$ intervals of latitude and longitude

gaps between frames. This meant that the entire sky beyond $\sim 18^{\circ}$ Sunward of the pitch axis, excepting a small region in the anti-solar direction, was observed during each 102-minute orbit. Post-processing involved projecting the individual camera exposures back onto the sky to form a complete sky map, and then presenting the result in a manner such as that shown in Fig. 3. This Hammer-Aitoff projection (e.g., Leighly 1955) is suitable for observing the entire sky; additional projections used for SMEI sky maps are further discussed in Sect. 3.1, and two of these are shown in Fig. 4.

When the Sun approached within about $7^{\circ}$ of the edge of Camera 3's FOV, a protective shutter closure prevented sunlight from directly reaching the CCD (Eyles et al. 2003). On average, this closure reduced the sky coverage of Camera 3 to about $60 \%$ of the full coverage around an orbit, as through the year the Sun moves relative to the spacecraft's orbital axis. During normal operations the shutters of Cameras 1 and 2 did not close.

For most of the year, apart from a short period close to the summer solstice, the Sun lay within the $18^{\circ}$ exclusion zone for Camera 3 . Within this exclusion zone is the region typically observed by coronagraphs, although no coronagraph has yet observed farther than $7^{\circ}$ out from the Sun. (The HI-1 heliospheric imager on board STEREO was the first instrument to observe in the angular gap between $7^{\circ}$ and $18^{\circ}$, but it only observes within $\pm 35^{\circ}$ of the ecliptic plane.)

Because each SMEI sky map is a built-up "image" assembled from a series of exposures, every location on the map corresponds to a slightly different time throughout the orbit. A SMEI sky map is therefore not a "still" frame, such as is acquired from the STEREO/HIs or most coronagraphs, but rather a time-varying scan across a 102-minute orbit. The short time that SMEI viewed a given location in the sky created sharper images of transients compared with the more conventional staring optical systems. This feature enabled more accurate measurements of their features (e.g., their leading edges). However, because a whole SMEI sky map does not provide an average at a single instant in time, the corresponding motion of heliospheric structures must properly be taken into account to calculate accurate structure locations.

\subsection{Sky Projections}

Figure 4 shows examples of two kinds of SMEI sky maps we have used, following some post-processing. Note that in Fig. 4 the gridlines show position angle and elongation, while in Fig. 3 they show latitude and longitude. 
a)

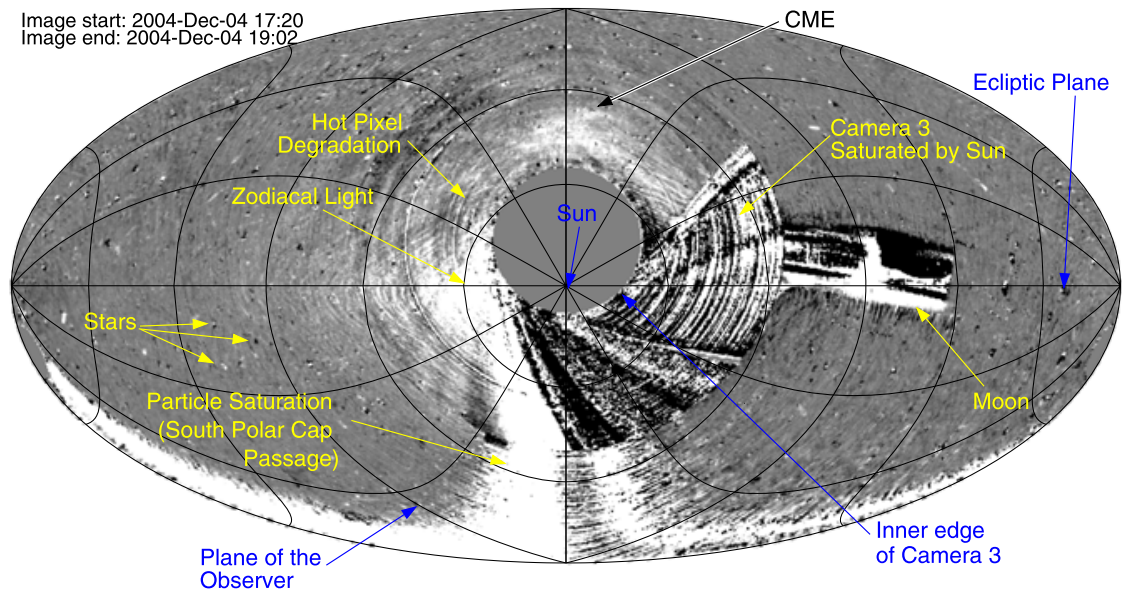

b)

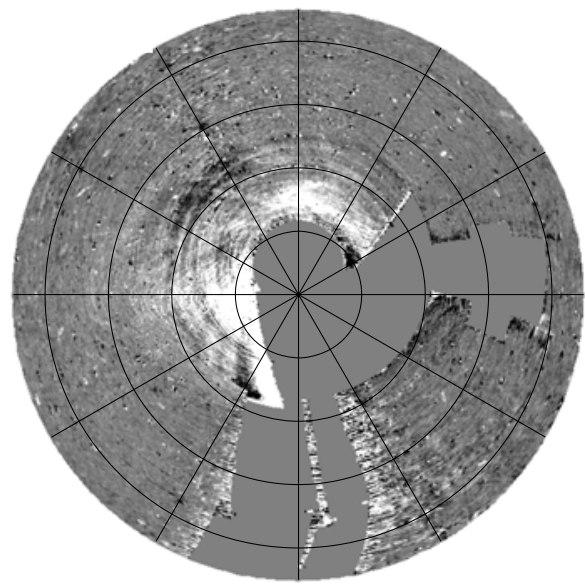

Fig. 4 Examples of a complete SMEI sky map in Sun-centered ecliptic coordinates. (a) Hammer-Aitoff projection (from Tappin et al. 2012), with various features noted. (b) The Sunward portion of the same map in an equal-radius "fisheye" projection, extending out to elongations $\varepsilon<135^{\circ}$ from the Sun. The circles (in (b)) and near-circles (in (a)) mark $30^{\circ}$ contours in $\varepsilon$, whereas the more radial lines coming out of the Sun mark position angle (increasing counterclockwise with zero at ecliptic north). A number of features are labeled, and in panel (a) the sources of noise have been explicitly left unmasked to show their appearance in a SMEI sky map. In panel (b) the noise sources have been masked out (solid gray). These sources are the Sun, Moon, Venus, dark charge and electronic bias, and excessive particle noise

Figure 4a shows a SMEI sky map as a Hammer-Aitoff projection, referred hereafter simply as an Aitoff projection. Such an equal area projection can be difficult to interpret at a glance because it folds the entire sky into a two-dimensional projection. When this representation became conceptually difficult, the zenithal equidistant projection, also known as and hereafter referred to as a "fisheye" projection, was also often used. Figure $4 \mathrm{~b}$ shows a fisheye version of the same sky map as Fig. 4a. Fisheye projections are easier to understand at a glance since a radial from the Sun is a straight line, but these have the disadvantage that they do not cover the entire sky (we rarely use them beyond $135^{\circ}$ elongation, as the distortion becomes too large). These maps were made in heliocentric ecliptic coordinates, 
i.e., the equator of the map is the ecliptic plane, and longitudes are measured relative to the Sun.

The features labeled in blue in Fig. 4a identify important locations in the Aitoff map. The Sun lies at the center of the map and every surrounding point is measured as a function of elongation $\varepsilon$ (angle between the Sun-Earth vector and the vector from the Earth through the measured point) and position angle, which is the angle from due ecliptic North (upwards) rotating in a counterclockwise direction. Linear distance (as opposed to angular distance) is meaningless in this kind of map, for reasons that are discussed in Sect. 4.1.2. The gridlines therefore indicate $30^{\circ}$ contours of elongation (those gridlines centered at the Sun) and position angle (those directed radially out from the Sun). The location of the observer (the Earth) itself does not appear in this map, but rather, the plane perpendicular to the SunEarth line at the location of the Earth intersects the celestial sphere at the $90^{\circ}$ elongation contour. Anything observed at larger elongations than the $90^{\circ}$ contour is therefore behind the observer when looking towards the Sun. When interpreting the propagation of transients such as CMEs, these move approximately radially outward from the Sun, and pass the $90^{\circ}$ elongation contour when they have arrived at the Sun-centered sphere containing the Earth. A large spherical transient could therefore also strike the Earth when it reached this contour on the map. Further, since the fisheye projections are cut off at elongations $>135^{\circ}$, the anti-solar direction (to the left and right of the Aitoff map) does not appear in those maps.

\subsection{Factors Affecting SMEI Image and Sky Map Quality}

The SMEI cameras required a high sensitivity and wide dynamic range in white light, as the desired CMEs and other structures have an intensity maximum of only a few $\times 10^{-14} B_{\odot}$ where $B_{\odot}$ refers to the mean surface brightness of the Sun (see Sect. 6.1). Moreover, since the surface brightness of these features rapidly diminishes with distance from the Sun, the dynamic range of the instrument must span at least four orders of magnitude. Furthermore, the cameras had sources of background and other factors affecting image quality, ranging from instrumental (hot pixels, glare, scattered light), to environmental (particle impacts during the SAA and auroral oval passages), to astronomical (Moon, planets, zodiacal light, individual stars and galactic background). Figure 4a shows many of these, labeled in yellow.

\subsubsection{Instrumental Factors}

Significant instrumental noise was caused by "hot pixels". These are CCD pixels that show a significantly-elevated dark current and in some cases may flip between normal and hot states. This was particularly problematic in Camera 3, which was operating at temperatures typically between $-15^{\circ} \mathrm{C}$ and $0{ }^{\circ} \mathrm{C}$ (depending on the time of year), substantially above the design temperature of $-30^{\circ} \mathrm{C}$. This caused many pixels to be hot, and some flipped on timescales of less than an hour. Cameras 1 and 2, which were running close to or below the design temperature, also developed some hot pixels later in the mission, but these were not sufficiently numerous to noticeably impact their performance when analyzed with other than "quick-look" data processing (see Sect. 3.3).

Annealing returned many of the hot pixels to their normal state. For this process, the CCDs were typically heated to about $+60{ }^{\circ} \mathrm{C}$ to $+80^{\circ} \mathrm{C}$ for a few hours, using the decontamination heaters that were closely coupled thermally to the CCD chip package. Because of its numerous hot pixels, Camera 3 was annealed more often than the other two and at a higher temperature $\left(+80^{\circ} \mathrm{C}\right)$. In spite of the annealing efforts, hot pixels gradually accumulated throughout the mission, contributing to the dark charge noise and degrading the image 
quality. By around 2006 Camera 3 images had degraded to the point where only the brightest transients could be directly observed in unprocessed or "quick-look" sky maps, and by the end of the mission in 2011 Camera 2 had also noticeably degraded, but not enough to compromise its observation of CMEs. Each camera displayed an increase in dark charge through the course of the mission due to orbital radiation flux, but the most significant degradation by far was in Camera 3. To put this into some perspective, we note that SMEI operated well beyond its nominal design life of 3 years.

A second contribution from the instrument we call "glare". This is a light contribution reaching the image plane of the CCD through a scattering path other than the optical-design path. In the case of SMEI, mechanical design constraints resulted in paths other than those corresponding to the normal FOV via which sunlight could reach the CCD. This light could be modeled and thus largely eliminated from the final SMEI maps. Buffington et al. (2005) identify one scattered-light source of this glare not removed by the modeling, which could be remedied in a future imager.

\subsubsection{Environmental Factors}

The polar Earth-orbit of Coriolis produced additional sources of noise, primarily from energetic particle CCD impacts. The two most troublesome orbital regions for this are the SAA, which is essentially an extension of the Van Allen radiation belt to low altitudes, and the auroral-oval zones, where the ionosphere is directly exposed to energetic particle precipitation from the solar wind. When especially numerous, energetic particles can saturate the cameras, creating the well-known "snow storm" effect that is common with coronagraph images as energetic solar particles impact the instrument. In the auroral zones the particles are most likely to be electrons: these were first collimated by the baffles and then scattered by the main mirror in the SMEI optics to reach the CCD directly. In the SAA the particles are mostly energetic protons which penetrated the instrument housing. At low to moderate fluxes, the affected CCD pixels are identified in the SMEI analysis and excluded from the summing to make the final maps, but with high fluxes this is no longer possible. The result is saturation such as is visible in the lower parts of the sky maps in Fig. 4.

\subsubsection{Astronomical Factors}

SMEI was capable of detecting stars (at a $3 \sigma$ threshold within a square-degree bin) down to 10th magnitude in a single sky map (Buffington et al. 2006a). The resulting numerous stars therefore dominated the FOV of each camera. In principle, these could be individually subtracted from each sky map, or even from individual data frames before forming a sky map. In practice, stars only down to 6th magnitude were individually subtracted, since SMEI's large point-spread function (PSF) causes significant overlap when considering stars fainter than this. The instrument was designed to spread the PSF across multiple pixels in an attempt to mitigate possible sub-pixel variations in detector sensitivity (Eyles et al. 2003; Jackson et al. 2004). Typical examples showing the variation in the PSF across the image plane are shown in Fig. 5. Star fitting and removal are further discussed in Sects. 3.3.1 and 4.2.4.

Saturation by the Sun of Camera 3 (as seen in the bottom-right part of Camera 3 in Fig. 4) occurred when the spacecraft attitude was such that the Sun's location approached too closely to the FOV of the innermost camera. Closing of Camera 3's shutter (saturated region) removed some of this, but not all. Note in Fig. 4a that the $18^{\circ}$ exclusion zone is substantially offset from the location of the Sun, which here approaches the edge of the exclusion zone. 


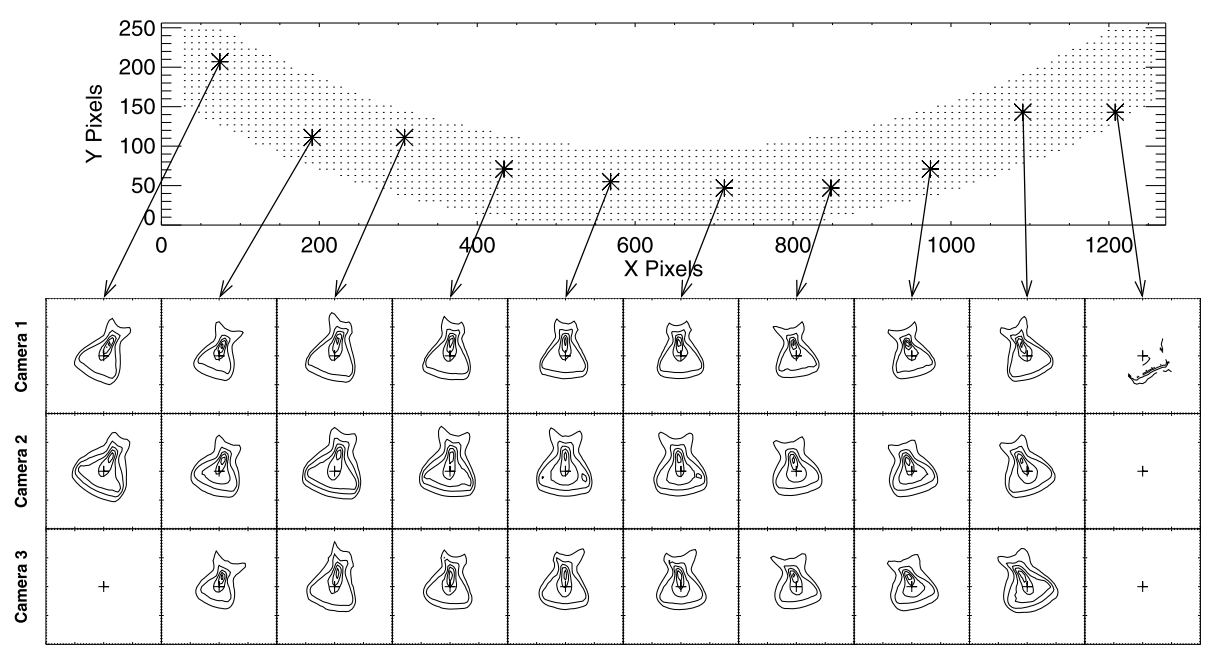

Fig. 5 Illustration of the variation of the SMEI point-spread function across the detectors. The upper panel shows the imaged strip of the sky projected onto the CCD detector, the dots indicate the locations at which PSFs were determined. For those locations indicated by asterisks, the PSF for each of the three cameras is shown in the lower panels. Each PSF is shown in a panel of $41 \times 41$ pixels. The centroids of the patterns are indicated by the plus signs

Another large saturating feature across Camera 2 in Fig. 4a is the Moon (labeled). The Moon posed a significant problem for SMEI observations, as it completely saturated large portions of the sky even when it was only a small crescent near to the Sun. While nothing could be done to remove the saturation effects of the Moon, it was a known and predictable entity, and easily flagged as bad data (it has been masked in Fig. 4b). Likewise, the brightest planets (Venus, Jupiter) saturated SMEI in a predictable manner and so they could also be flagged, while the other planets could be treated as "stars", and subtracted.

The final source of astronomical background was the zodiacal light, also known as the F-corona. For SMEI this light dominated the sky, particularly close to the Sun around the ecliptic plane. When observed at the sensitivity of SMEI, the zodiacal dust band and annual variations due to the geometry of the cloud were significant, so simple zodiacal light models could not be used. A more complex model was required, involving an accurate characterization of the solar off-axis contribution to the background. Alternatively, such contributions can be reduced by subtracting a background derived directly from the data. This method included both running differences (subtracting the previous-orbit's map), and subtracting a three-day median sky map as used in Fig. 4. This latter method, as shown in the portion of Fig. 4a labeled Camera 3 (which shows the results of the background subtraction), does not entirely remove the zodiacal light. A further alternative used in the 3-D reconstructions applied a Gaussian temporal filter to remove most of the long-term (2-week) zodiacal light variation that was not directly removed in the analysis. Section 3.3 discusses the data processing pipelines that were developed by SMEI team members.

\subsection{Data Processing}

The net response of the three cameras differed: the responses of Cameras 1 and 3 were matched to those of Camera 2, each by a multiplicative factor (Buffington et al. 2007). Camera 1's factor was 0.97 and Camera 3's factor was 0.93 . Further, due presumably to 
CCD radiation effects through the mission, the net response to stars diminished by $1.6 \%$ per year (Buffington et al. 2007), while tracking portions of sky along the Galactic disk by $1.0 \%$ per year, and the bright center of the Galaxy by $0.6 \%$ per year. For most purposes, a time-dependent gain correction of $1 \%$ per year has proven adequate (Buffington et al. 2009a).

Several techniques were developed in order to reduce the noise factors discussed in Sect. 3.2. The three most commonly used of these, each developed by a research group within the SMEI team, are summarized below.

1. The Air Force Research Laboratory (AFRL) pipeline was developed pre-flight but evolved over the first two years of the mission. It was made specifically for the purpose of producing "quick-look" maps for rapid space weather forecasting (see Sect. 5.1). Aitoff sky maps and fisheye projections were produced, correcting for bad pixels, cosmicray contamination, detector dark charge, flatfields and attitude jitter. An AFRL zodiacal model was adapted to the SMEI passband and a mean stellar background map was created to remove the bright stars and diffuse galactic emission. The model worked well at removing the large-scale zodiacal light for solar elongations greater than around $30^{\circ}$, but nearer the Sun proved problematic. A secondary mean sky map was calculated using the first three of the four previous orbits (skipping the orbit immediately prior to the observed orbit and neglecting any with significant contamination) and subtracted as a background. The processing pipeline originally took around 90 minutes to import a telemetry file and produce the processed sky maps, but two years into the mission streamlining reduced the processing time to 45 minutes. Figures $9 \mathrm{~b}, 13$, and 18 show examples of SMEI data processed via the AFRL pipeline. Additionally, the AFRL pipeline had procedures for mitigating flipping pixels, and removing bright single-frame transient phenomena such as space debris.

2. The UCSD pipeline (Hick et al. 2005) imported the SMEI data into a UCSD database and then performed a conditioning to remove the CCD electronic offset and temperaturedependent dark current. Next, an "indexing" process placed individual frames onto a $0.1^{\circ}$ high-resolution sidereal grid using the known spacecraft pointing information. At this indexing stage, further conditioning removed the bulk of the effects of high-energy particle hits (Sect. 3.2.2), some of the more fast-moving space debris inside the field of view (Sect. 5.2), and pixels with sudden discrete state changes (flipping pixels, see Sect. 3.2.1). From these sidereal maps, bright stars, background stars, and a zodiacal cloud model were removed. Figures 11a, 14 and 16 show examples of SMEI sky maps from the UCSD pipeline.

3. The National Solar Observatory (NSO) pipeline, developed by the University of Birmingham (UK) and NSO groups converted raw data from SMEI into the sky maps, examples of which are shown in Figs. 4 and 12a. The procedure is discussed in detail in Appendix A of Tappin et al. (2012). Briefly, once the background contributions from each frame (including CCD electronic offset and dark charge, together with glare) were removed and gain correction applied, known bright artifacts (the Sun, Moon, Venus, etc.), hot pixels and pixels affected by particle hits were identified and flagged. A star removal technique was applied (discussed in the next section), using the same star list as was used in the UCSD pipeline. Further background subtraction was then applied to remove the zodiacal light and light from the ambient solar wind. This final background-removal stage could be performed in several ways, most commonly using a running difference or a three- or six-orbit base subtraction where the median of previous and following orbits was subtracted. 


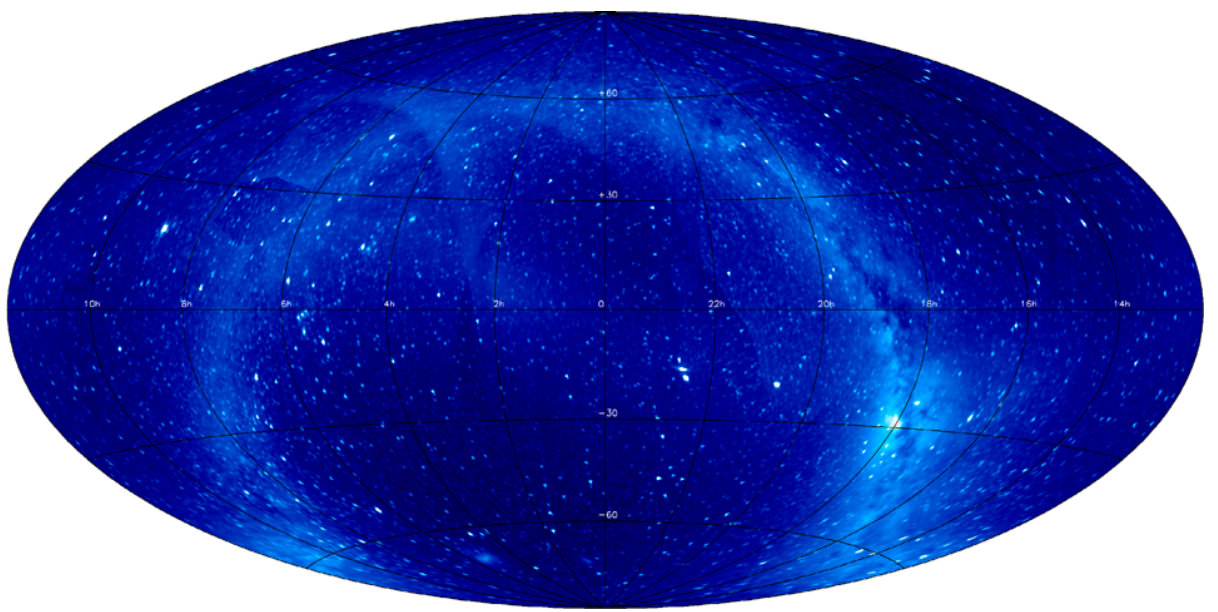

Fig. 6 Complete star map obtained from the sum of many SMEI maps made over some months. The coordinates for this map are right ascension, declination (equatorial)

\subsubsection{Star Removal}

As mentioned in Sect. 3.2.3, several methods were developed by the various team members to remove the stars from SMEI data. Two of these removed stars after the formation of single-orbit sky maps, while a third removed them directly from the SMEI image frames. Figure 6 shows a complete sky star map summed over many orbits. For the construction of this map, orbits were chosen such that the Sun and the Moon did not appear in any of the maps. Glare was removed as much as possible. The bright feature in the map is the galactic center and the faint, almost circular, ring is the Milky Way. The faint diffuse feature going across the Milky Way in the upper-left quadrant is an artifact, which in principle could be removed by further extensive data processing. The constellations are recognizable, e.g., Orion on the equator to the left of center (near 6h right ascension).

1. Attempts were made to reduce the stellar background during the development of the AFRL pipeline, but such reductions were difficult to achieve given SMEI's irregular fishshaped PSF (see Figs. 5 and 19, and also figures in Eyles et al. (2003) and Hick et al. (2007)). A method was devised to "circularize" the PSF by convolving it with its transpose and "stretching" the result until a circularly symmetric PSF of about one degree in size was obtained. This mean PSF was built up from a six-month average of SMEI Camera 1 and 2 data, to minimize any residuals from the zodiacal model subtraction. However, this scheme left too many stellar residuals as the circularization preserved much of the high-frequency components in the PSF. Also, the background was never fully reduced to the noise level needed for CME detection. Since modeling and removing this stray light would have involved a long-term effort, this background method was eventually abandoned in favor of a faster product intended for forecasting (see Sect. 5.1).

2. The UCSD pipeline removed stars from individual sky maps. This involved fitting a standard PSF to known stellar positions for the brightest 6000 stars (Hick et al. 2007). The PSF was rotated according to the passage of the SMEI camera sweep over the sky, and according to the location of the star relative to the center of the camera frame. A leastsquares fitting procedure adjusted the stellar brightness, a planar background beneath the 
PSF, and the location of the stellar centroid position (Hick and Jackson 2004; Hick et al. 2007; Hounsell et al. 2010).

3. The NSO pipeline produced an empirical grid of PSFs at an array of locations within an individual frame's FOV, by summing the images around the point when a bright star was centered there; then a feature of that shape was subtracted from the locations of known bright stars (brighter than magnitude 6). Finally, the remaining faint stars and the galactic plane were subtracted using a two-year median map of the sky. This process was effective, but residuals remained for two main reasons: firstly, when stars were close together the fitting of the PSF shape could be somewhat compromised; and secondly, as the mission progressed, there was some excess scattering in the brightest stars making their PSFs somewhat wider than the above grid of PSFs predicted.

\section{Science Results: Overview and Highlights}

SMEI routinely imaged almost the entire sky in white light at better than a 10th stellar magnitude level in a square degree, producing up to 15 sky maps per day. This unique dataset provided many opportunities for scientific advancements, not just in solar physics and space weather, but also in auroral physics and in astrophysics. In this section we review the many scientific advancements that arose from SMEI. We divide this section into two parts: primary, where we report on work related to CME and related science; and additional, where we briefly review the other scientific advancements.

\subsection{Primary Science}

The SMEI primary mission was focused upon CMEs. SMEI immediately proved able to detect heliospheric CMEs out to and beyond $90^{\circ}$ elongation, and continued to observe them right up until its last day of operation (a CME was in fact observed on the morning of the shutdown). A total of 391 CMEs have been confirmed throughout the mission. This amounts to less than $10 \%$ of the CMEs observed by the Large Angle Spectroscopic Coronagraph (LASCO, Brueckner et al. 1995) and cataloged during the same period: compare, for example, with 9955 CMEs from CDAW and 4462 from CACTus. SMEI lists have appeared in the papers by Webb et al. (2006) and Howard and Simnett (2008), and a complete catalog is available at http://smei.ucsd.edu/.

\subsubsection{Coronal Mass Ejections}

In the first SMEI results paper, Tappin et al. (2004) reported on a CME seen in May 2003. This was the first major geoeffective CME observed during the SMEI mission, and is shown in Fig. 7. The CME is observed as two arcs outside of the shuttered areas, one large moving northward and a smaller one moving toward the southeast. Figure 7 also shows the quality of the SMEI quick-look sky maps early in the mission, but this quality improved as the SMEI team's processing techniques developed (compare, for example, with Fig. 4). The distance-time curve for this event, which was extrapolated backwards to the Sun, is shown in Fig. 8a, and matched well in timing with two CMEs that were launched from the Sun early on 28 May, and observed as "halos" by the LASCO C3 instrument. Two forward shocks were measured by the Advanced Composition Explorer (ACE, Stone et al. 1998) spacecraft on 29 May, and a major geomagnetic storm began towards the end of the same day. This combination of SMEI sky maps, coronagraph images, and in-situ datasets proved to be a 


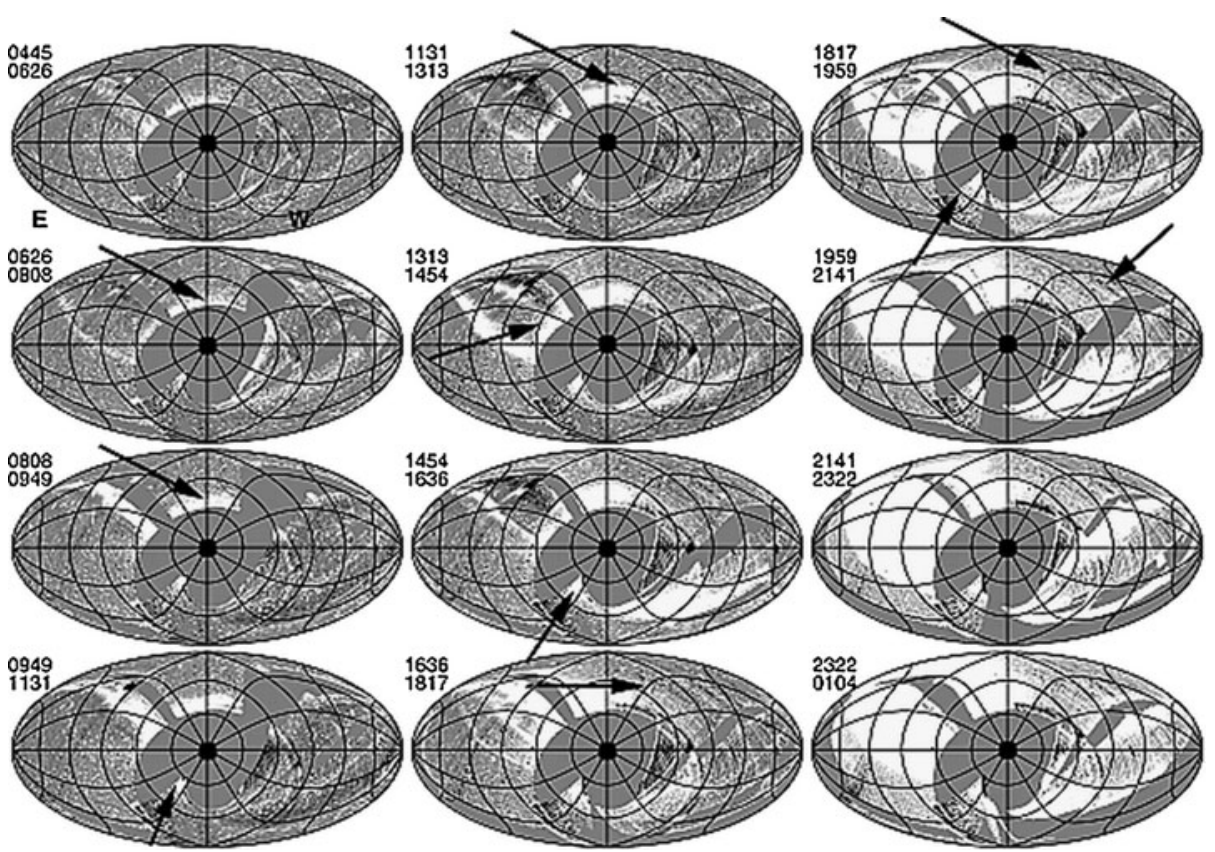

Fig. 7 Early SMEI Aitoff sky maps showing the first geoeffective CME of the SMEI mission (from Tappin et al. 2004). The sequence shows almost a complete day (29 May, 2003) of maps and moves from top to bottom, left to right, with the start and end times provided in the top-left of each map. The CME appears as two arcs (arrowed) in the SMEI sky maps: a large arc moving northward (upwards); and a small arc moving towards the southeast (down-left)

powerful tool, and enabled for the first time almost continuous visual tracking of CMEs from their solar origins through the heliosphere to the Earth. We now believe, but cannot say for certain, that the CME component most likely observed by SMEI was the sheath in most cases, probably comprised of built-up solar wind material ahead of the underlying CME magnetic structure. There have, however, been some studies of specific SMEI CMEs for which a more detailed study of their components was possible. Jackson et al. (2006), for example, identified both the sheath and prominence components of the Halloween CME and measured their masses. Early papers reporting SMEI observations of CMEs include Howard et al. (2006), who tracked 20 forward shock-producing CMEs through SMEI back to their solar origins, Jackson et al. (2006), who employed a 3-D reconstruction technique on the Halloween (2003) CMEs and the surrounding solar wind conditions, and Webb et al. (2006), who summarized the SMEI CME measurements for the first $1 \frac{1}{2}$ years of operation.

Towards the end of 2006, the twin STEREO spacecraft (Kaiser et al. 2008) were launched carrying, among other instruments, their Heliospheric Imager (HI) ensembles (Eyles et al. 2009). The HIs provided an excellent dataset to complement SMEI, as their images were free of many of the noise sources in Earth orbit that affected the SMEI data (e.g., magnetospheric energetic particles, hot pixels, Moon), but the HIs are limited in their fields of view. They therefore do not see those parts of the sky at large angles from the Sun to the north and south of the ecliptic. Also, they obtain their images by long exposures, so they produce somewhat "blurred" images of transient phenomena. Combining the two instruments for a single CME observation was first done by Webb et al. (2009a) (Fig. 9) and Jackson et al. (2009a). By comparing sky maps where a long temporal base is removed with 

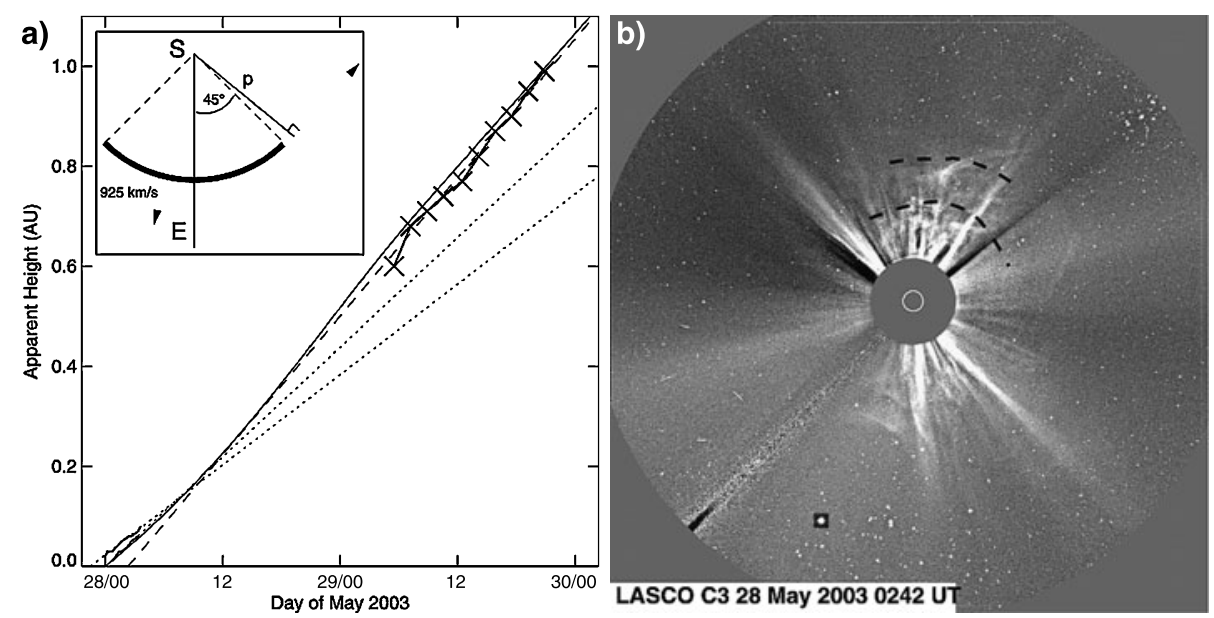

Fig. 8 (a) Distance-time plot of the May 2003 SMEI event from Tappin et al. (2004). Units of elongation have been converted to distance in AU using the Point $\mathrm{P}$ approximation $(r \sim \sin \varepsilon)$, i.e., it is the perpendicular distance from the LOS intersecting the leading edge assuming the measured CME feature is a large spherical shell centered on the Sun (insert, upper left). The dashed line shows the extension back to the Sun and forward to beyond $1 \mathrm{AU}$. The solid curve is the extrapolation for a structure extending to $45^{\circ}$ from the Sun-Earth line and traveling at $925 \mathrm{~km} / \mathrm{s}$, shown schematically in the insert. (b) LASCO C3 coronagraph image of the associated CME, which is actually two CMEs launching in quick succession (black dashed arcs). Their distance-time plots are also shown by the dotted lines in panel (a)

"running-average" maps, the Jackson paper shows that structures with large gradients are emphasized in the latter, while the former clearly show that more dense and extensive CME structure trails the high-gradient features. Other comparisons followed (e.g., Howard and Tappin 2009a, 2010; Jackson et al. 2010b, 2011c; Webb et al. 2013).

Other SMEI work with CMEs includes comparison with interplanetary scintillation (IPS) radio data (e.g., Tokumaru et al. 2007; Jackson et al. 2007a, 2007b, 2011b; Bisi et al. 2008), comparison with in-situ data (e.g., Tappin et al. 2004; Howard et al. 2006; Jackson et al. 2006, 2008, 2011c; Tappin and Howard 2009a; Webb et al. 2009b, 2013), and space weather forecasting (Sect. 5.1). Tappin (2006) analyzed one CME that was tracked to $~ 5 \mathrm{AU}$ using LASCO, SMEI and Ulysses data, and the non-radial motion of CMEs was reported by Kahler and Webb (2010).

\subsubsection{Three-Dimensional Reconstruction of CMEs and Results}

Section 3.1 mentions that linear distance is not measured in SMEI data. This is true of all heliospheric imagers, as the large angular span in the FOVs of such imagers prevents the application of the standard Point-P and small-angle $\sin \varepsilon \sim \varepsilon$ assumptions that are applied to coronagraph images. Applying these two approximations yields simply $r$ (AU) $\approx \varepsilon$ (radians).

These approximations break down at the larger angles from the Sun observed by heliospheric imagers. The "plane of the sky" ceases to be a plane at these angles, but instead the locus of all points closest to the Sun for all lines of sight forms a sphere with its diameter as the Sun-observer line. Vourlidas and Howard (2006) termed this the "Thomson surface" and theoretical explorations of this concept are discussed at length by Howard and Tappin (2009a) and Howard and DeForest (2012a). In the early days of SMEI, in 


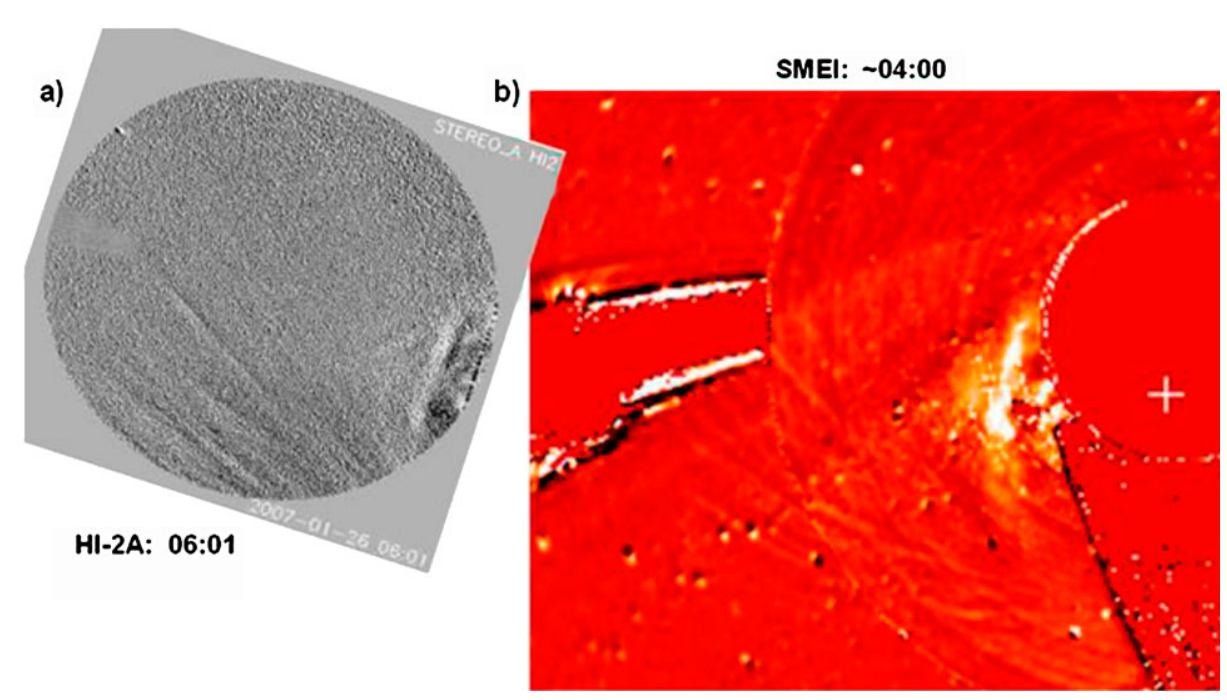

Fig. 9 Comparison of the fields of view between (a) HI-2A and (b) SMEI using data obtained during the early months of the STEREO mission (Webb et al. 2009a). These were obtained around the same time on 26 January 2007. The HI-2A image has been scaled and rotated to approximate its size and orientation relative to the SMEI FOV. Several common features appear in both views, including a group of CMEs to the right of each image and fragments of the tail of comet McNaught towards the south. These appear as streaks across the bottom of each image. The location of the Sun is indicated by the + in the SMEI image, but it does not appear in the HI-2 image as it is outside the instrument's FOV. The masked feature to the left of the SMEI image is the Moon, which also does not appear in the HI-2 image

one of the display types, elongations were converted into distance using the plane-of-thesky (Point-P) approximation (see, for example, Tappin et al. 2004; Howard et al. 2006; Webb et al. 2006). Beyond around $30^{\circ}$ additional three-dimensional information is needed. Out to around $45^{\circ}$, the so-called Fixed- $\Phi$ approximation (Sheeley et al. 1999) was applied (Kahler and Webb 2007). Fixed- $\Phi$ assumes the measured structure is a single point, and applies spherical geometry including a propagation direction to convert elongation to distance (Howard et al. 2007). Howard and Simnett (2008) demonstrated that the elongation-time profile can change dramatically with changing propagation direction, as shown in Fig. 10.

At angles larger than around $45^{\circ}$ it was found that a complete 3 -D treatment was unavoidable. This is because at large angles the structure of the CME begins to affect the location where the lines of sight cross the structure. This is important as distance-time measurements obtained from the AFRL and NSO pipelines were always made on the apparent leading edge of the observed structure (see Howard 2011, for details).

A more complete 3-D treatment of the CME location and density distribution has been addressed with three different approaches:

1. Empirical reconstruction of the solar wind and transient structure by the conversion of SMEI brightness units into density via tomographic techniques;

2. Direct comparison of SMEI maps with synthetic sky maps produced from existing solar wind magnetohydrodynamic (MHD) models;

3. Empirical reconstruction of transients using leading-edge measurements.

Treating these in order, empirical reconstruction of the solar wind extends a technique that originated with Helios data (Jackson and Leinert 1985) and was applied to IPS datasets (e.g., Jackson et al. 1997, 1998, 2011c; Kojima et al. 1998; Hick and Jackson 2001; 
Fig. 10 Elongation-time $(\varepsilon-t)$ plots for a single point moving in the equatorial plane, traveling with a speed of $1000 \mathrm{~km} / \mathrm{s}$, measured relative to an observer at the Earth looking at the Sun (Howard and Simnett 2008). Curves show changes in the $\varepsilon-t$ plot as the longitude of the trajectory moves from near the Sun-Earth line $\left(0^{\circ}\right)$ to the solar $\operatorname{limb}\left(90^{\circ}\right)$

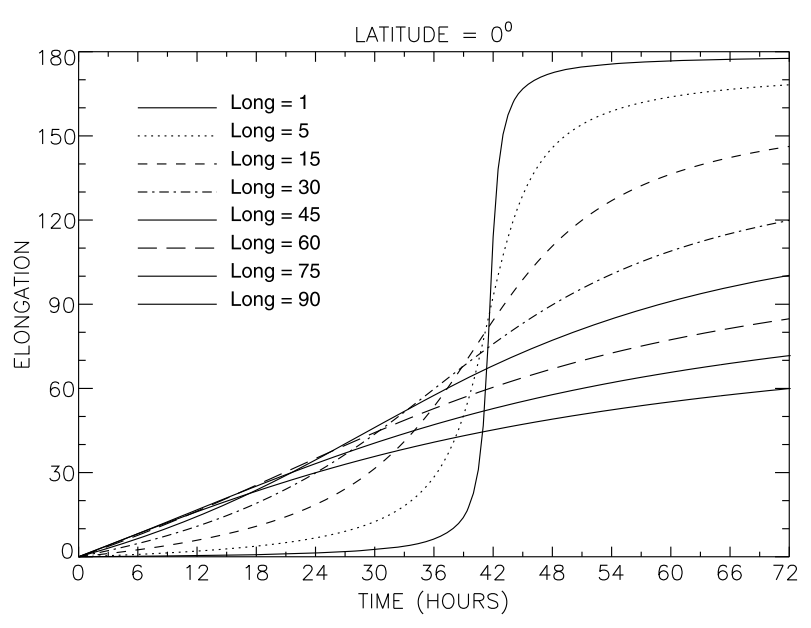

Bisi et al. 2010, and reviewed in Jackson et al. 2011b). This technique applies the known basic physics of the heliosphere and Computer Aided Tomography (CAT) techniques to heliospheric brightness viewed remotely. Briefly, for the time-dependent tomography used in the SMEI analysis, the solar wind is reconstructed by projecting it outward from a source surface, assuming radial outflow, and conservation of mass and mass flux to obtain the threedimensional solar wind speed and density. SMEI sky maps, where a long-term base is subtracted, and heliospheric in-situ and IPS velocity measurements, are used to improve the solution for the density and velocity of material everywhere along the lines of sight.

Yu et al. (2005) and Jackson et al. (2006) describe a volumetric display system which has proven useful for visualizing reconstructed SMEI data, and determining volume, mass and energy of CMEs. These values were compared with coronagraph observations for specific structures within the CME (Jackson et al. 2006, 2008). Figure 11a shows a CAT reconstruction of a CME observed by SMEI in January 2007 (from Webb et al. (2009a) and Jackson et al. (2009b)). Other publications involving this technique include Hick and Jackson (2004), Jackson and Hick (2004), and Dunn et al. (2005).

The UCSD 3-D time-dependent reconstructions provide CME mass estimates that compared well with coronagraph measurements of individual portions of CMEs (Jackson et al. 2006, 2008, 2011a). In these analyses, various portions of the CME response were measured (the shock sheath, the erupting prominence material), and compared with equivalent portions of the CME in coronagraph observations. In the analysis of the 28 October 2003 CME, Jackson et al. (2006) showed that the prominence material of the CME is elongated in the direction of the Earth, and approximates the loop-like magnetic field structure measured in-situ at Earth for this event (Jensen et al. 2010; Jackson et al. 2011a). The SMEI reconstructions agreed well with in situ density measurements at Earth and STEREO (e.g., Jackson et al. 2008, 2010b, 2012).

Model comparison involves the processing of simulated SMEI sky maps using existing solar wind and interplanetary transient models, for direct comparison with actual SMEI data. The two models that are commonly used with SMEI for this purpose are HAFv2 (Fry et al. 2001) and WSA-ENLIL + CONE (Odstrĉil et al. 2004). Both models begin with an MHD solar wind model, constructed using empirical measurements of the lower solar atmosphere. In summary, HAFv2 treats the CME as a kinematic disturbance or pulse in the solar wind medium, determined by solar flare and Type II radio burst data for the boundary conditions. WSA-ENLIL + CONE introduces the CME as an additional parcel of plasma, the density, 
a)

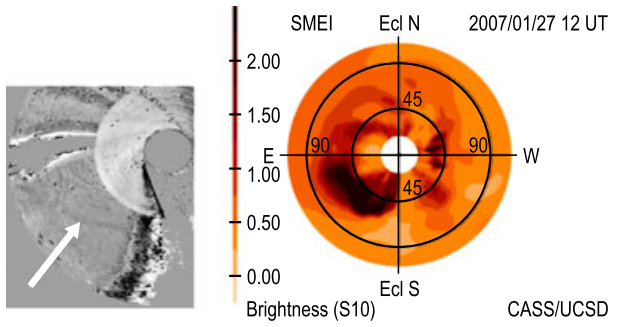

b)
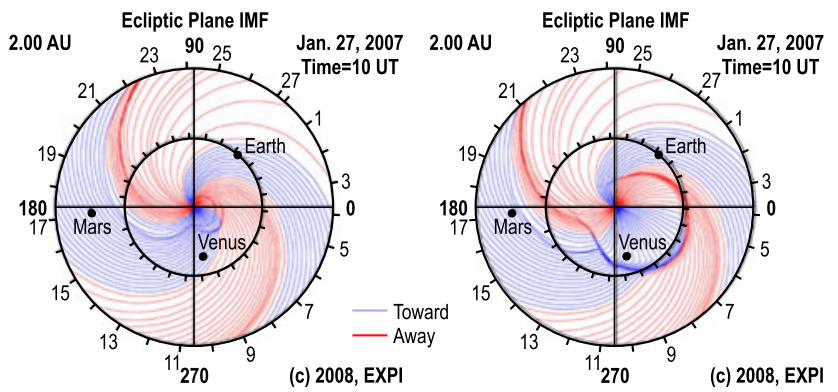

c)

ENLIL-25 medres WSA-1.6 NSO 2007-01-27 02:00:05 2007-01-24 +3.08 days OMercury OVenus OEarth OMars $\square$ Messenger $\square$ Stereo_A $\square$ Stereo_B Ecliptic Plane W90 LAT $=5.6^{\circ}$
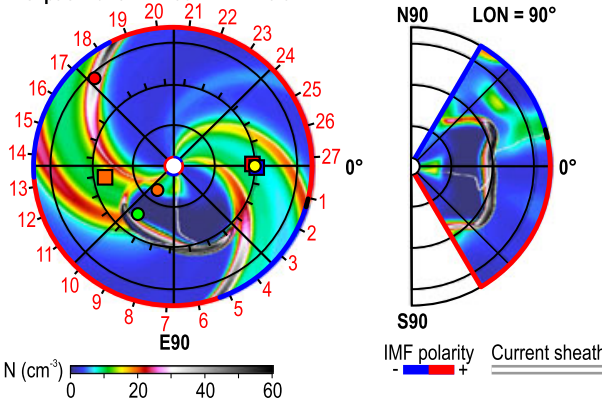

IMF polarity Current sheath

d)

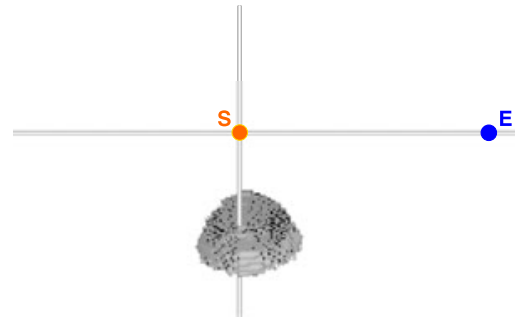

Fig. 11 Results for the three-dimensional reconstruction of CMEs using methods employed throughout the SMEI mission. These are for a CME observed on 27 January 2007. Panels (a)-(c) are from Webb et al. (2009a). (a) SMEI fisheye sky map (left) and fisheye tomographic reconstruction (right) using the CAT technique (see text) at 12 UT. The white arrow shows the CME in the SMEI sky map. (b) HAFv2 solar wind disturbance reconstruction at 0 UT on the previous day (left) and at $10 \mathrm{UT}$ (right), looking down onto the ecliptic plane and showing the orientation and polarity of interplanetary magnetic field lines. (c) WSA-ENLIL + CONE MHD solar wind injection reconstruction on 02 UT, showing the density of the solar wind and looking down onto the ecliptic plane (left) and along the north-south meridian (right). (d) TH model reconstruction, looking down onto the ecliptic plane from the north. In panels (b)-(d) the location of the Sun and Earth are shown, and all four models show considerable agreement, with the reconstructed CME moving along the eastern perpendicular to the Sun-Earth line (i.e., a limb CME) and somewhat to the south 
structure and kinematics of which are determined by coronagraph measurements. Both models propagate the created disturbance out to $1 \mathrm{AU}$ and beyond, enabling direct comparison with SMEI data. Figures $11 \mathrm{~b}$ and c show results for each model for the January 2007 CME. Publications involving HAFv2 comparison with SMEI include Howard et al. (2007), Sun et al. (2008), Jackson et al. (2008) and Webb et al. (2009a), while those comparing WSAENLIL + CONE include Bisi et al. (2008), Webb et al. (2009a, 2013), and Jackson et al. (2010b).

Leading-edge comparison is based on the assumption that the leading edge of a CME is an easy feature to measure in SMEI data, and follows investigations showing that the location of the apparent leading edge of a CME does not vary significantly with large variations in a simple simulated CME's density (Howard and Tappin 2008). This enables a simplified approach using geometry alone to govern the structure and trajectory of the measured CME. The Tappin-Howard (TH) Model (Tappin and Howard 2009a) compares a hypercube of simulated leading edges with actual leading edge measurements from SMEI, using a convergence routine involving a genetic algorithm. A basic structure of a Sun-centered spherical arc is assumed, distorted from the perfect sphere with a distortion function. The result is a set of parameters from the simulation that best describes the structure and orientation of the leading edge (latitude and longitude location, and width, distortion and speed), along with uncertainty contours describing the "goodness" of each parameter convergence. Figure $11 \mathrm{~d}$ shows the TH reconstruction for the January 2007 CME. Publications involving the TH model on numerous CMEs include Tappin and Howard (2009a), Howard and Tappin (2009b, 2010), Howard and DeForest (2012a), Howard et al. (2012), and Webb et al. (2010a, 2010b, 2013).

These 3-D reconstruction results demonstrate that information is available in heliospheric imager data that is unavailable in coronagraph data. With careful analysis, 3-D structural and kinematic information can be extracted directly from heliospheric imager data without the need for auxiliary datasets. The 3-D reconstruction of near-Earth features is a major milestone of the SMEI mission. A number of complementary 3-D reconstruction techniques have subsequently been developed for use with $\mathrm{HI}$ data, including, for example, the HarmonicMean method (Lugaz et al. 2009) and forward modeling (Xiong et al. 2011, 2012).

\subsubsection{CME Discoveries}

Along with the identification and tracking of CMEs, SMEI data enabled the confirmation of two classes of CME, which were suspected to be present but not previously confirmed by coronagraphs.

The first class is now known simply as erupting magnetic structures (Lyons and Simnett 2001). Work comparing in-situ interplanetary forward shocks with halo CMEs (Howard and Tappin 2005a, 2005b; Gopalswamy et al. 2010) identified a small number of shocks that could not be associated with CMEs. Studies with SMEI enabled a more reliable association with coronagraph CMEs, as it closed the large gap between the in-situ datasets at $1 \mathrm{AU}$ and coronagraphs near the Sun. It also enabled the study of non-halo CMEs. CMEs observed by SMEI but not by LASCO were reported by Simnett and Kahler (2005), by Simnett (2005), and finally by Simnett and Howard (2006) and Howard and Simnett (2008). These studies all found that about 10-15\% of observed SMEI CMEs could not be associated with a LASCO CME. They were attributed to erupting structures in the corona with insufficient mass to be observed by coronagraphs (so-called "invisible CMEs"). Presumably the magnetic structure had accumulated enough material ahead of it (the snowplow effect) to be observable by the time it reached the FOV of SMEI. 
The second class of CME confirmed by SMEI are termed V-arc CMEs. Kahler and Webb (2007) described five CMEs that were outward concave in shape (i.e., with their "nose" pointed towards the Sun). They were attributed to trailing edges of CME flux ropes or leading CME edges sheared by solar wind speed and density gradients at the heliospheric current sheet.

\subsubsection{Corotating Interaction Regions}

Corotating interaction regions (CIRs, Smith and Wolfe 1976) arise from the interaction between a fast-flowing column of solar wind material (e.g., from a coronal hole) and the surrounding slow solar wind, which causes a compression along the interface. These effects are strongest near the solar equator. The disturbance produces waves, which eventually develop into a shock at large distances from the Sun.

Because of their dominance far from the Sun it was thought that SMEI would regularly observe CIRs, especially in the declining and minimum solar phases during the latter years of the originally-planned mission. However, only occasional CIR activity was observed by SMEI. The first one was reported by Tappin and Howard (2009b), using observations in November 2008 (near solar minimum). It was identified by its slow relative speed and location at elongations beyond $100^{\circ}$, where CMEs are almost never observed. Figure 12 shows a SMEI observation of this CIR, along with elongation-time plots from the HIs on STEREO-A and -B. Another was identified in tomographic reconstructed data, interacting with a CME (Jackson et al. 2010b). CIRs are now detected in white light regularly in HI data, with the signature to the east of the Sun-spacecraft line easily identified using elongationtime "J-maps" (e.g., Sheeley et al. 2008; Rouillard et al. 2008; Tappin and Howard 2009b; Howard et al. 2012).

\subsubsection{Interplanetary Shocks}

Jackson et al. (2010c, 2011c) reconstructed the density enhancements behind shocks and compared them with in-situ measurements at Earth, and where possible at STEREO-A and -B. In Jackson et al. (2011c), the enhancements are compared with IPS velocity measurements, with densities from Wind and CELIAS, and also with two different in-situ data sets from ACE. The shocks in these analyses at Earth have been confirmed in the CELIAS Proton-Monitor (Hovestadt et al. 1995) shockspotter, routines that measure the increase in temperature, speed, density and magnetic field change at the shock front in the heliosphere, and are also mapped by SMEI density and IPS velocity measurements in UCSD 3-D reconstructions. These analyses show that the SMEI brightness calibration (Buffington et al. 2007) is as accurate as the in-situ measurements for large scale features in the solar wind. There is substantial evidence that shock sheaths are not continuous enhancements in the solar wind in front of a CME but are "spotty" features as viewed in the remote sensing measurements (Jackson et al. 2009a, 2011c), and confirmed in-situ (Jackson et al. 2010c, 2011c). As reviewed briefly in Jackson et al. (2011b) for the 28 October 2003 CME, the density enhancements behind interplanetary shocks can be measured to determine their mass and energy content.

Jackson et al. (2010b, 2011c) compare SMEI observations with shock sheaths measured in-situ. These studies aimed at determining the extent to which shock sheaths include dust swept up from the inner heliosphere. Enabled by the calibrated SMEI surface-brightness data, these analyses gave a null result for shock dust contamination down to the limit SMEI could determine this effect. These analyses also highlight the large columnar density differences (as large as $50 \%$ ) that are determined from the different in-situ monitors that measure the same shock sheaths at the L1 Lagrange point. 
a) Image start: $2008-\mathrm{Nov}-24$ 12:11

Image end: 2008-Nov-24 13:52

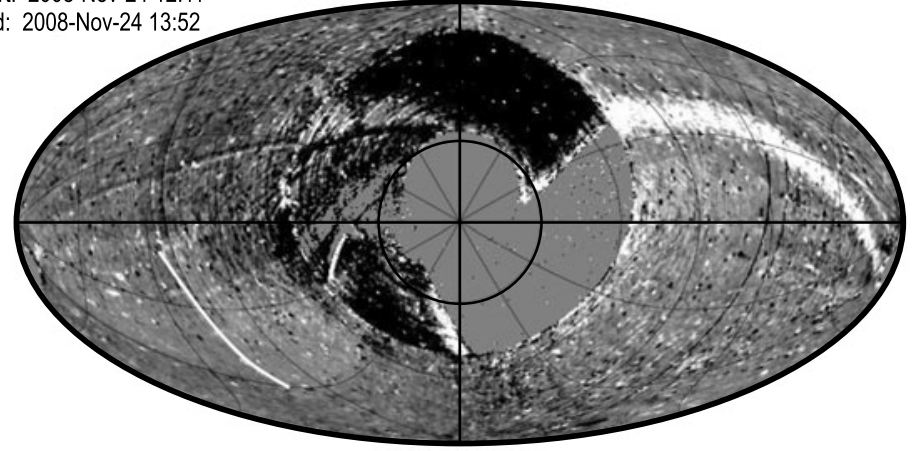

b)

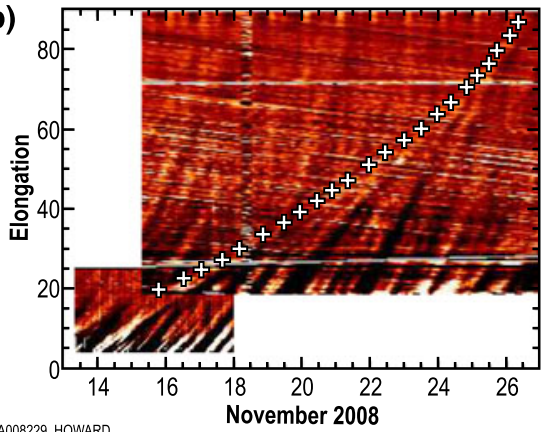

c)

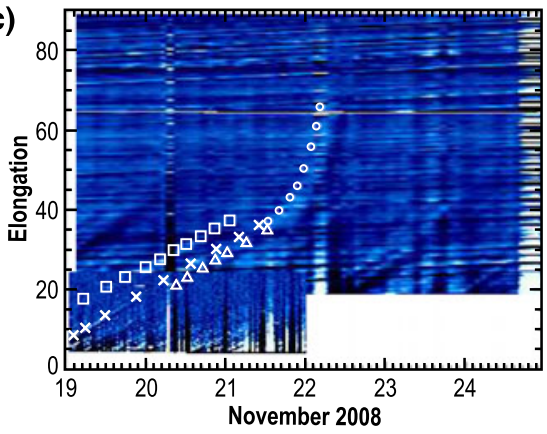

Fig. 12 SMEI and HI views of a CIR observed in November 2008 and reported by Tappin and Howard (2009b). (a) SMEI Aitoff map from 24 November, with the CIR indicated with a white arc to the south-east (bottom-left). The bright arc to the right of the map is due to energetic particle saturation. (b) and (c) STEREO/HI J-maps showing the CIR as it evolves through the FOV of (b) HI-A and (c) HI-B. A J-map is a $\varepsilon-t$ map constructed by stacking a sequence of views and taking a slice through a particular position angle (see Sect. 5.1.1). The different symbols in panels (b) and (c) denote leading-edge measurements of separate structures

\subsection{Additional Science Topics}

A variety of additional scientific advances were made using SMEI. Many of these were accomplished early in the SMEI mission, but studies continue to the present. In the following sections we review the more significant advances accomplished using this unique dataset.

\subsubsection{Forbush Decreases}

Frequently in ground-based neutron monitors, a decrease in counting rate is observed that can last for several days. Such decreases are known as Forbush decreases (Forbush 1954), and they are believed to be caused by transient, enhanced local magnetic fields near the Earth modulating incoming galactic cosmic ray scattering and convection (Wibberenz et al. 2002; Cliver et al. 2003). Simnett and Kahler (2004) and Kahler and Simnett (2005) demonstrated how SMEI could be used as a forecasting tool for Forbush decreases. Using a high-latitude neutron monitor, they identified 22 Forbush decreases from March 2003 until May 2005. All were associated with both a transient in SMEI and a CME observed by LASCO, and most were halo CMEs (see Sect. 5.1). Using elongation-time plots and comparing these 


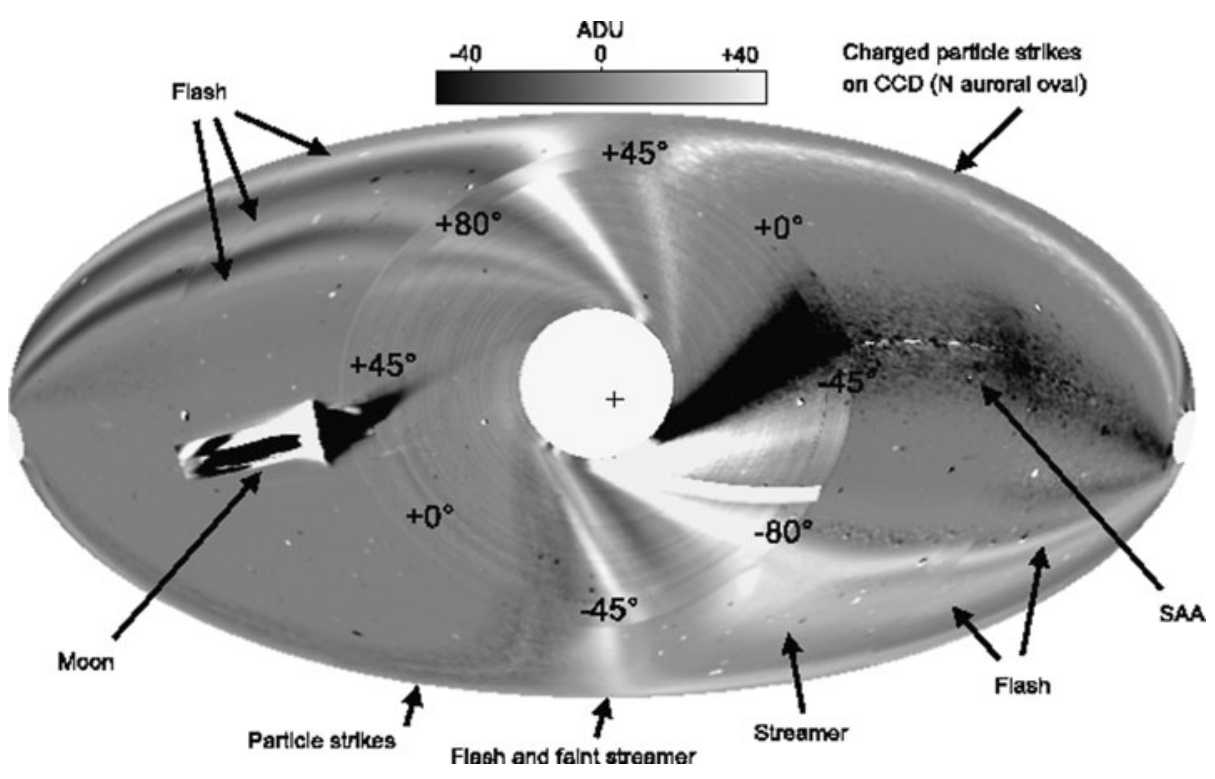

Fig. 13 SMEI Aitoff sky map on 30 October 2003 showing several features, including the two classes of aurorae: "flash"; and "streamer" (Mizuno et al. 2005). The numbers around the outer edge of the Camera 3 field of view are the geographical latitude of Coriolis at the time of that part of the image

with LASCO CMEs, a prediction of the occurrence and onset time of Forbush decreases could be estimated.

\subsubsection{High-Altitude Aurora}

While aurorae have been observed at altitudes reaching $1000 \mathrm{~km}$ (e.g., Størmer 1955), these are a rare phenomenon. Since SMEI viewed only above its $842 \mathrm{~km}$ altitude, aurorae were seldom expected to be observed. This assumption proved to be false, with aurorae often saturating the SMEI cameras, most significantly during times of enhanced geomagnetic activity (i.e., when $\mathrm{K}_{\mathrm{p}}>4$ ).

Mizuno et al. (2005) reported on a survey of aurora during the first year of SMEI's operation. They divided the auroral signatures observed by SMEI into two categories: (1) a "flash" having bright regions where all three cameras were briefly illuminated simultaneously $(<1$ to 3 minutes); and (2) a "streamer" with one or more filaments curving asymptotically with increasing time toward the rearward direction of the satellite. These both are diffuse features, easily distinguished from particle strikes that affect only one or a few pixels on a given CCD. Figure 13 shows AFRL-processed SMEI map from the Halloween (2003) event with the two categories of aurora.

During SMEI's first year, a total of 823 SMEI orbits with flashes and 219 with streamers were observed: an average of around one auroral signature every five days. A good correlation with geomagnetic activity indices was found. Flashes occurred as the spacecraft passed directly through a region brightly illuminated by the aurora, and the streamers as SMEI viewed columns of luminous material some distance away from the spacecraft. 

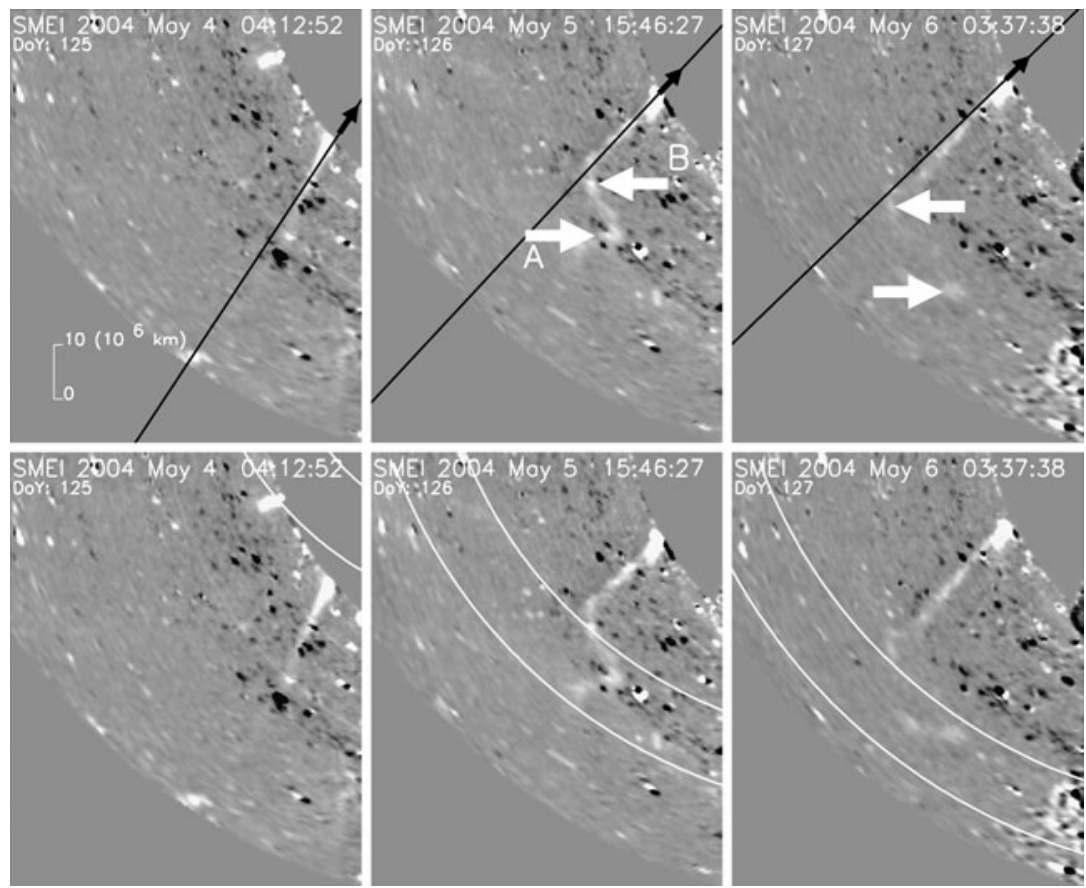

Fig. 14 SMEI fisheye images of a disconnection of the tail of comet NEAT during 4-6 May, 2004 (from Fig. 2 of Kuchar et al. 2008). Three images are shown, (left) before, (middle) during, and (right) after the disconnection. The black line indicates the radial direction to the Sun drawn through the position of the comet and the white arrows indicate two separating fragments: labeled A and B. Two CME arcs were observed to pass by the comet around the time of disconnection, they are highlighted by the white arcs in the bottom row of images, which otherwise are the same as the top row

\subsubsection{Comets}

SMEI's wide FOV enabled comet-tail observations that were previously unfeasible. SMEI provided the first observations of comet tail disconnection events at very large distances from the comet's nucleus (Kuchar et al. 2004, 2006). Kuchar et al. (2008) investigated six tail disruptions for comets NEAT and LINEAR, observed in 2004. Figure 14 shows images of one such disruption, which was noteworthy as it corresponded to the passage of a faint CME at the time of disruption. This shows that at least one disconnection event was likely caused by a CME, but the cause of the remaining five was uncertain: one suggested possibility was a heliospheric current-sheet crossing. The disconnection first appeared as a kink moving antisunward that eventually developed knots within the tail. The disconnected part became a circular arc roughly normal to the solar radial vector, which then moved away from the Sun. Later similar comet disconnection events were observed using HI data (Vourlidas et al. 2007; Clover et al. 2010) and, recently, Li et al. (2011) investigated an outburst of Comet 17P/Holmes using SMEI data.

In addition to complete disconnections, SMEI demonstrated that comet tails are sensitive indicators of variations in the solar wind: Buffington et al. (2008) described the buffeting of the tails as resembling smoke rising from a flaming torch. This work measured the radial speeds of smaller-scale tail disruptions as they moved away from the Sun, and compared them with in-situ and IPS solar wind speed measurements near the Earth. A poor correlation 


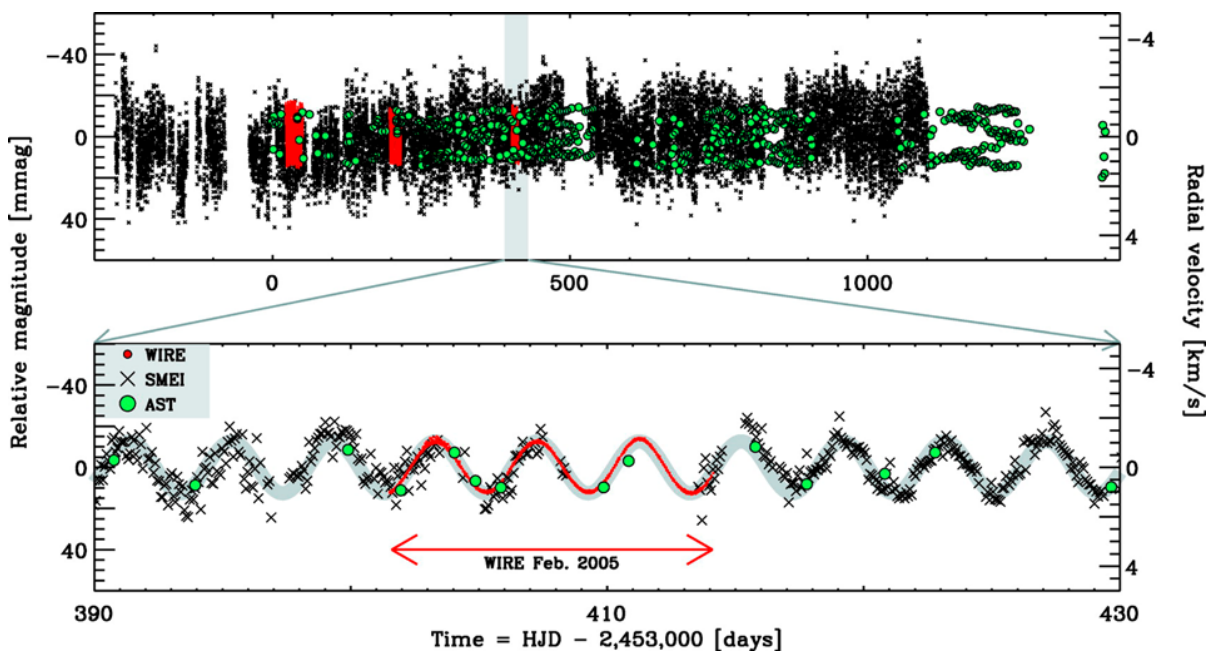

Fig. 15 Plot of the variability of the Polaris Cepheid variable using measurements from WIRE (red circles), AST (Automatic Spectroscopic Telescope) (green circles), and SMEI (crosses), over a $4 \frac{1}{2}$ year time frame (Bruntt et al. 2008). The bottom panel shows a close-up view of a small part of the time range, showing the good agreement between the measurements

was found, which was attributed to disparities between the sizes of the disruptions measured by the auxiliary datasets compared with those observed by SMEI.

This analysis of comet-tail motions shows that the solar wind outflow is chaotic and highly variable, and not the benign regular outward motion of a quiescent plasma (Jackson et al. 2012). Such comet-tail observations, using heliospheric white-light imagers, are important because they can provide solar wind information closer to the Sun than where spacecraft, with their in-situ measurements, have ventured to date.

\subsubsection{Variable Stars}

Stars brighter than 6th magnitude observed by SMEI were individually fitted and removed for the CME analyses. The fitted brightness dataset has proven to be a valuable resource for stellar variability studies. Penny (2006) compared ground and SMEI measurements of the Polaris Cepheid variable. Bruntt et al. (2008) studied $4 \frac{1}{2}$ years of data on Polaris, comparing ground spectroscopic data with photometric measurements from both the WIRE (Wide Field Infrared Explorer (Bruntt 2007)) star tracker and SMEI. Contrary to previous studies that revealed a decrease in the amplitude of oscillation over time, this work revealed an increase in amplitude by around $30 \%$ over this time. This finding challenged earlier claims that Polaris was moving towards the so-called instability strip. Figure 15 shows the amplitude variability measurements using WIRE and SMEI.

Other work using SMEI to study variable stars includes Penny et al. (2003), presenting early work on stellar brightness and crowding, and Bruntt et al. (2006), who studied eclipsing binaries. Also Spreckley and Stevens (2008) studied Polaris and provided valuable light curves for further stellar work, and Tarrant et al. (2008a, 2008b, 2008c) reported on oscillations in $\beta$ Ursae Minoris and $\gamma$ Doradus, and asteroseismology of red giant stars, respectively. Finally, SMEI searched for corresponding optical signatures for gamma-ray bursts (Buffington et al. 2006a). However, there were no detections at the sensitivity limit of SMEI. 


\subsubsection{Novae}

SMEI provided a substantial increase in available data for novae, particularly on the rising phase, which previously was poorly characterized. Hounsell et al. (2010) presented light curves from the rise phase of four novae with unprecedented temporal resolution, enabling the accurate identification of the epoch of initial explosion, the duration of pre-maximum halt, any presence of secondary maxima, and the speed of decline of the initial light curve. For one event, this work identified the precise timing of the onset of dust formation, which led to an estimation of the bolometric luminosity, white dwarf mass, and object distance. These authors also emphasized the fact that many bright novae, particularly those with short duration outbursts, are currently overlooked or are observed after the rise, considering that most instruments do not view a given portion of the sky all the time.

\subsubsection{Zodiacal Light and the Gegenschein}

In Sect. 3.2.3, we regarded the zodiacal light as a background and discussed the means by which it was removed for the study of CMEs. However, with star removal and a carefully designed subtraction of instrumental background, it was possible to study the background scattered sunlight, the zodiacal light, from measurements using SMEI. Zodiacal light is brightest near the Sun. Studies of zodiacal light using SMEI include Buffington et al. (2006b, 2009b). SMEI R-band measurements of the zodiacal dust bands were obtained by Penny (2006) and Buffington et al. (2006b). Buffington et al. (2009a) present formulae describing the zodiacallight surface brightness in the anti-solar hemisphere; extension of this parameterization to the other hemisphere is still currently under development.

A portion of the zodiacal light, the Gegenschein, is a diffuse brightening of the night sky centered on the anti-solar point in the sky. It is caused by sunlight back-scattered from dust in the interplanetary medium, and is sometimes distinguished from the rest of the zodiacal light because the high angle of reflection of the sunlight allows a more efficient scattering process. An enigmatic issue involves the detailed distribution of the scattering dust relative to the Earth, which requires an accurate characterization combined with a scattering phase function. Buffington et al. (2009a) used SMEI data to characterize the Gegenschein (see Fig. 16), describing the brightness distribution, shape and variation with time, and confirming the commonly-held view that the brightness distribution has a peak at the anti-solar point that rises above the broader background.

\section{Operational Aspects}

Although SMEI was a proof-of-concept mission, it was designed not only to demonstrate the scientific quality of CME measurement and tracking, together with providing an onorbit validation of the instrument design with its many novel features, but also to investigate whether heliospheric imaging data could be used to improve the quality of Air Force space operations, particularly space weather forecasting. The potential of SMEI to improve space weather forecasting was well demonstrated and moreover it proved useful in observing Earth-orbiting satellites. These two contributions of SMEI to space operations are reviewed in this section. 
Fig. 16 Plot of the Gegenschein and surrounding regions of the F-corona from Buffington et al. (2009a), in units of anti-solar latitude and longitude

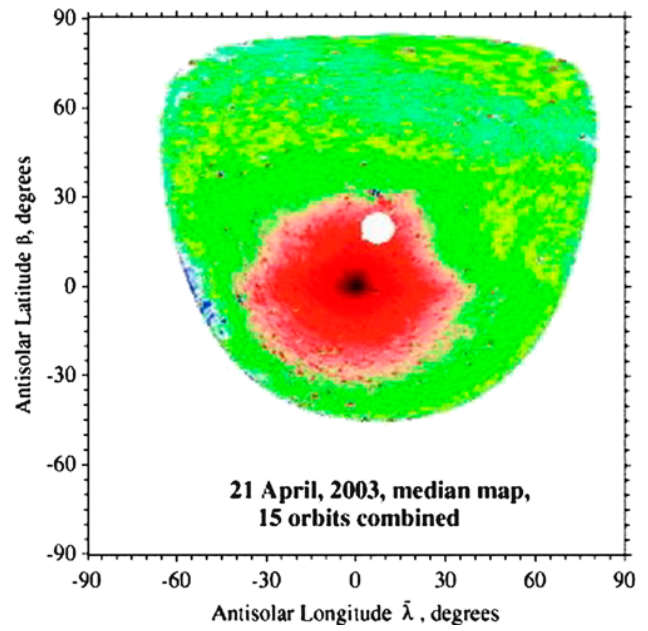

\subsection{Space Weather Forecasting}

When SMEI was launched, it was well established that those CMEs observed by coronagraphs available at the time, that were most likely to be Earth-impacting were the "halo CMEs". A halo CME has a considerable component directed along the Sun-Earth vector, and, hence, appears in a near-Earth coronagraph as a halo surrounding the occulting disk (Howard et al. 1982, 1985). Such CMEs can be near-sided or far-sided, and while it is impossible to determine the difference between the two solely with coronagraphs, near-sided CMEs can be identified using heliospheric imagers, whereas the far-sided CMEs fade in intensity at very large distances from the observer. Near the Earth, geomagnetic sudden commencements are often caused by the arrival of an interplanetary shock, typically driven by a CME (e.g., Taylor 1969; Ogilvie and Burlaga 1969; Dryer et al. 1972). Also, highlystructured magnetic flux ropes within CMEs, magnetic clouds, are known to interact with the Earth's magnetosphere, causing the most intense geomagnetic storms (e.g., Wilson 1990; Marubashi 2000; Echer et al. 2005). With the exception of IPS radio measurements and data from Helios and occasional observations by other in-situ spacecraft, the almost 1 AU gap in space between the coronagraph's FOV and the near-Earth in-situ measurement was not observed prior to the advent of SMEI. Predictions of CME arrival time, speed and geoeffectiveness were therefore limited to models that extrapolated the evolution of the CME using data from coronagraphs viewing close to the Sun (Sect. 4.1.2 reviews some of these), or short timescale $(<\sim 1$ hour) alerts from in-situ measurements at L1. SMEI bridged the gap between the Sun and the Earth, and enabled timely comparisons with the forecasting models. This improved the accuracy of impact probability, arrival time and speed calculations. However, because one could typically not identify and track a CME reliably until it was closer than $0.5 \mathrm{AU}$ to the Earth, predictions often became available only a day or so before the CME actually arrived at Earth (e.g., Webb et al. 2009b).

The first attempts to use SMEI for space weather forecasting were made by Howard et al. (2004, 2006). They associated post-facto interplanetary shock measurements near the Earth with LASCO halo CMEs by projecting SMEI distance-time plots forward to the shock and backward to the halo CME. By forward-projecting the SMEI distance-time plot, an estimate of the CME's arrival time at 1 AU could be made, which was compared with the in-situ shock measurements. Figure 17 shows the accuracy of some of these early predictions. 


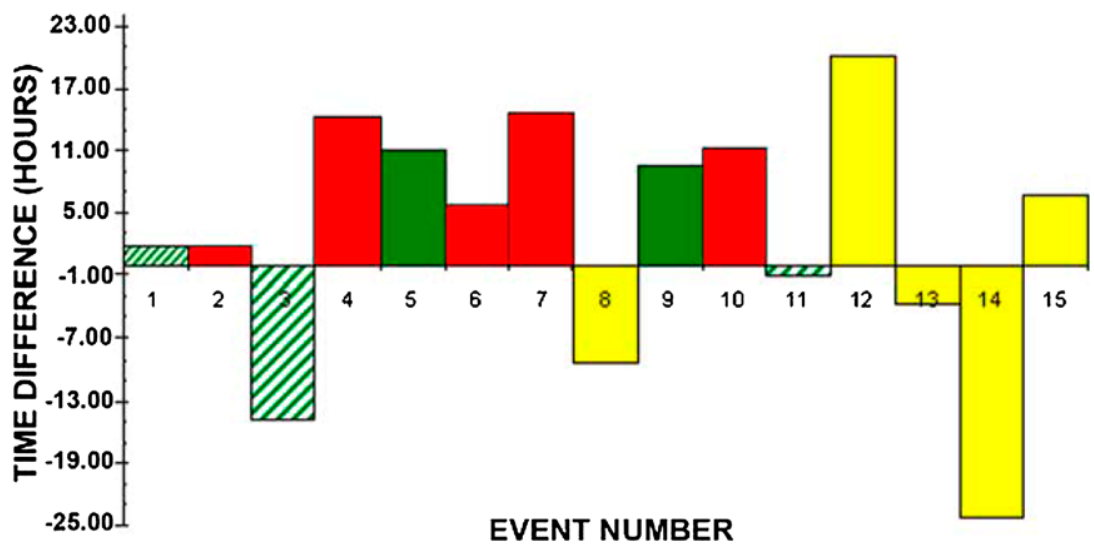

Fig. 17 Histogram showing the difference between the predicted arrival time and actual arrival time for 15 geoeffective CMEs measured in the early years of SMEI by Howard et al. (2006). Colors denote the intensity of the associated geomagnetic storms: $r e d=$ large $\left(A_{p} \geq 80\right.$, Dst $\left.\leq-150\right)$; yellow $=$ medium $\left(A_{p}=60-80\right.$, Dst -150 to -80$)$; green $=$ small $\left(A_{p}=30-60\right.$, Dst -80 to -60$)$. Cross-hatched bars indicate storms with no sudden commencement (Webb et al. 2009b)

AFRL considered the demonstration of the capability of forecasting as a primary goal of the SMEI mission. As stated in Sect. 3.3 the explicit goal of the AFRL processing pipeline was the production of a computationally-rapid sky map construction system for near-realtime space weather forecasting. To this end, they created a processing pipeline that met the AFRL requirement to deliver SMEI products (including maps) within six hours of the original data having been obtained on the spacecraft (and not from the time transmitted). SMEI had too high a data latency to actually do such forecasting (although it was successful for a few events). AFRL developed a SMEI measurement tool for the purpose of providing forecasts to the Air Force Weather Agency (AFWA) and a simplified user-friendly SMEI "point-and-click" tool was created for AFWA and used in conjunction with conventional forecasting techniques. Although the tool could use any of the SMEI image products, the quickly-produced fisheyes were the primary data input. The tool would allow the user to interactively measure and track the front of a CME as it propagated through differenced sky maps. The set of elongation/time measurements were used to predict $1 \mathrm{AU}$ arrival times assuming a simple constant launch speed and trajectory from the Sun. If the computed trajectory was within $30^{\circ}$ of the Sun-Earth line, the CME was considered as traveling Earthward. Studies of events indicated that Earthward-directed CMEs generally showed geoeffective behavior within 3-6 hours of the predicted 1 AU arrival times. This tool evolved over the lifetime of the mission to become a simpler graphical user interface (GUI) based IDL tool for general users. Figure 18 shows the GUI interface with an elongation/time prediction plot based on user measurements.

Eventually, all three of the 3-D reconstruction techniques discussed in Sect. 4.1.2 were applied to the space weather forecasting effort. UCSD provided regular all-sky tomographic reconstructions using SMEI data as they became available (see http://smei.ucsd.edu/ smeidata.html); predictions of CME arrival times employing HAFv2 and WSA-ENLIL + CONE could be adjusted using SMEI sky map comparisons to make "mid-course corrections" (Webb et al. 2009b). Tappin and Howard (2009a) and Howard and Tappin (2010) devised a tool ("TH reconstructions", see Sect. 4.1.2) that enabled a speedy evaluation of CME leading edges and issued arrival time and speed forecasts as each new SMEI sky 

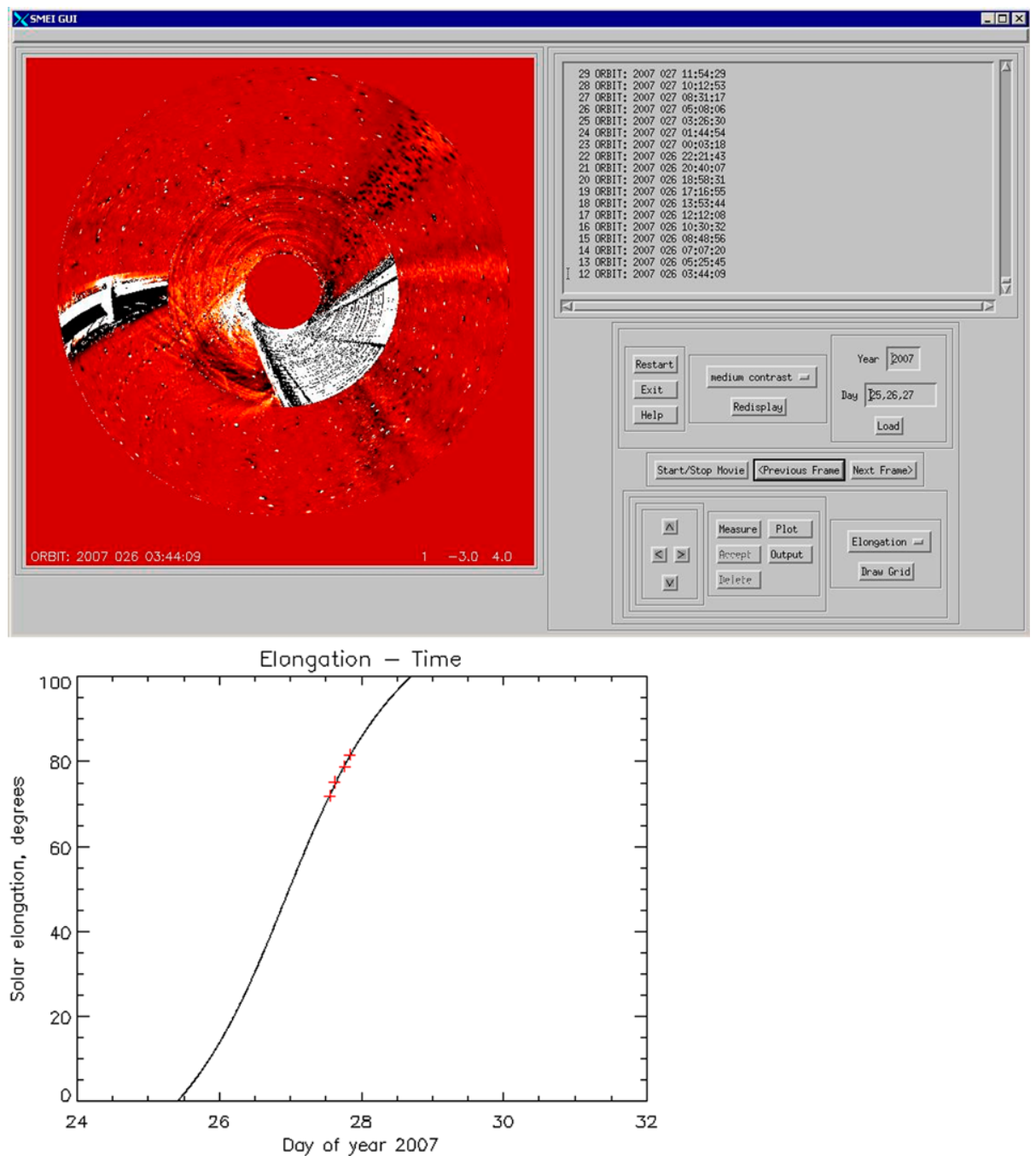

Fig. 18 (Top) the IDL-based GUI for measuring elongation-time points for a series of "quickly" produced fisheye maps. (Bottom) a prediction plot showing the user-measured elongation-time points $(+)$ and the propagation path calculated by the SMEI tool

map was formed. Using this technique, Webb et al. (2009b) reported an RMS accuracy of 10 hours measured against the previous best case of 11 hours and Howard and Tappin (2010) showed an accuracy as good as two hours for some cases (Webb et al. 2010a). SMEI therefore demonstrated that heliospheric imager data are a highly important addition to the space weather forecasting toolbox. These SMEI observations, through 2005, were also used in a community study on geostorms, as reported by Zhang et al. (2007a, 2007b) and Richardson et al. (2006, 2007). 


\subsubsection{Autonomous Detection}

An important recent milestone in the application of heliospheric imagers for space weather forecasting is the autonomous detection of CMEs. Automatic detection is crucial for the establishment of an alert system, partly because it is helpful to remove the need for a user to monitor incoming data constantly, and partly because these all-sky maps can be difficult to interpret. Autonomous CME detection with coronagraph images has been available for a number of years, one of the first being CACTus (Robbrecht and Berghmans 2004; Robbrecht et al. 2009). Efforts toward the development of a similar technique with SMEI have proven difficult, partly because of noise in the SMEI data, and partly because linear distance-time plots, upon which CACTus relies, are not available from SMEI data since they are in units of elongation rather than distance (see Fig. 10). As noted in Sect. 4.1.2, with coronagraph data, elongation and distance are nearly proportional to one another.

Tappin et al. (2012) overcame this issue by parsing the SMEI data, that is, by breaking the $\varepsilon-t$ map into overlapping sub-windows each with a limited $\varepsilon$ range, then searching for continuous straight lines of different gradients between the sub-windows. While far from ideal, this technique did detect most of the CMEs that were previously known (Sect. 4.1), and several additional events were found that had been missed. This study has paved the way for future work on autonomous detection with other heliospheric imagers.

\subsection{Earth-Orbiting Spacecraft and Local Debris Tracking}

The panoramic imaging capability of SMEI resulted in the detection of a large number of Earth-orbiting objects (resident space objects-RSOs). SMEI observed RSOs at a rate of about one per minute. Often, they were far enough away that they drifted through the SMEI FOV against the backdrop of stars over several sequential four-second exposures, occasionally being observed for up to 10 minutes when orbital geometries were favorable. However, the majority of objects appeared as streaks that crossed the entire in-scan field in a single exposure. Roughly a third of all the extracted RSOs were not associated with objects whose positions were predicted using the publicly available two-line orbital element sets (Mizuno et al. 2011).

Kraemer et al. (2011) found that SMEI observed extended-emission plumes that fell into three categories: (1) uncontrolled fuel venting events-possibly explosions; (2) satellite launches; and (3) insertion burns. A total of 44 such phenomena were observed that, given the timing and geometry, constituted about $32 \%$ of the launches that fell within the detection window. Since satellite launches were typically detected 1-3 hours after launch, the observed phenomena may have been related to passivation of the upper stage (i.e., fuel dumps).

SMEI occasionally observed a "swarm" of objects that was manifest as a sudden, relatively brief occurrence of a large number of RSOs, many more than the normal rate, up to dozens detected in a four-second exposure (Mizuno et al. 2011). These swarms usually lasted for a few minutes and generally crossed the field of view in a consistent direction. Figure 19 shows an example of a swarm, a large number of objects abruptly entering Camera 3 from the sunward end (left). The likely explanation is that the swarms were created by space debris striking Coriolis and ejecting a large number of smaller objects, most likely from the spacecraft multi-layer insulation (MLI). Similar particle swarm events are fairly frequently observed with the SOHO coronagraphs and the STEREO HIs, and are attributed to interplanetary dust or micrometeorite particles striking the spacecraft MLI and causing the release of many smaller pieces of debris. 
Fig. 19 An RSO (Resident Space Object) swarm entering the sunward end of Camera 3 (each strip is a different time scan), occurring on 7 December 2006. The swarm appears as the bright streaks extending from the left edge of each strip. The trajectories of the objects are consistent with the objects being particles ejected from the top edge of the solar panel

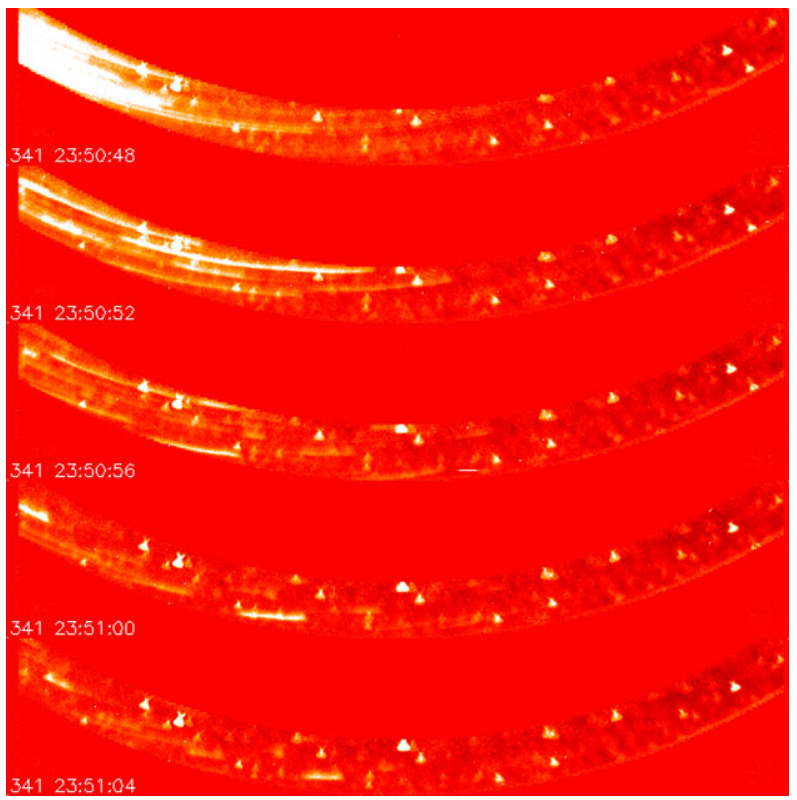

\section{Discussion}

As a proof-of-concept mission on a limited budget, SMEI was highly successful and some work is continuing on analysis of the data products. SMEI achieved its primary objective during the first month of mission operations, and went on to provide many further years of unique data. As the first example of a new class of imager, SMEI served as an invaluable pathfinder, and leaves an important legacy. Given that new heliospheric imagers are currently being proposed and in some cases being built, it is prudent to consider the lessons learned from SMEI to optimize the scientific returns from future missions. To conclude this review, we discuss those lessons and consider what we regard to be some of the important issues to be addressed in the design of, and analysis of the data from, these future heliospheric imagers.

\subsection{Lessons Learned}

The design concept of any heliospheric imager is strongly dependent on the overall mission concept, including such factors as deep-space versus near-Earth orbit, and low-inclination versus polar Earth orbits. As mentioned here and in the Introduction, and discussed at length in Jackson et al. (2010a), the Helios heritage had already provided important lessons that were employed in the SMEI design and were critical for delivering its success.

According to the specifications given in Table 1, the overall stray light background requirement for SMEI is $<10^{-15} B_{\odot}$. This demanding requirement was met in two parts. Firstly, an effective multi-stage baffle system provides rejection to a level of the order of $10^{-10} B_{\odot}$ before stray light can enter the camera entrance apertures (Buffington et al. 2003; Eyles et al. 2003). To achieve this performance, the SMEI cameras were mounted on the spacecraft so that the bright Earth was well below their FOVs, while the Coriolis orbit ensured that the Sun was within a zone of $\sim 18^{\circ}$ radius just beyond the end of the Camera 3 FOV. 
To provide the remaining $10^{-5} B_{\odot}$ of the stray-light specification, the subsequent optics minimized scattering by using two diamond-turned mirrors to focus the sky onto the CCD detector (and employed no other optical elements such as a protective window).

Because SMEI was a scanning system, in order to gather sufficient light over the orbital passage at a given sky location, the optical design had to be very fast, namely $f / 1.2 \times f / 2.2$. The small available CCD size resulted in severe mechanical constraints for placement of the optical elements, but a compromise in the design parameters yielded a PSF acceptable for achieving the desired $0.1 \%$ photometry (Eyles et al. 2003; Buffington et al. 1991).

Finally, a vane near the CCD performed part of the function of a Lyot stop in coronagraph optics, and all internal surfaces of the baffle structure and optic box were appropriately blackened (Eyles et al. 2003).

Separately, SMEI utilized the principle that by combining data over each orbit, the instrument should observe as much sky as possible. Besides the obvious value of tracking transients across larger distances and in all directions, this provides additional comparison with measurements from in-situ instruments located both near and far from the observer. Moreover, large sky coverage enables the best characterization of the background sky, which is then subtracted from the sky maps. SMEI's individual data-frame narrow FOV minimized CCD-saturated frames caused by the nearby Moon, which is a major consideration for nearEarth imagers with wide FOVs in both sky dimensions.

Several reports and presentations have already been published on lessons learned from SMEI, including Johnston et al. (2004), Jackson et al. (2007c, 2009a, 2010a), and Buffington et al. (2010). These articles highlight significant factors to consider, including the above, when designing a next generation of heliospheric imagers, wherever they may be deployed. In addition, they discuss design issues that emerged only after the launch of SMEI.

\subsection{SMEI Instrument Issues}

Several issues related to the design and in-orbit performance of the SMEI instrument need to be addressed for any similar instrument/mission in the future.

\subsubsection{CCD Operating Temperatures}

The design operating temperature of the CCDs used in SMEI was $-30^{\circ} \mathrm{C}$, to be achieved using passive radiators. In a Sun-synchronous polar orbit it is possible to orient radiators so that they have large view factors towards deep space, albeit also with significant view factors towards the Earth and spacecraft structures, which reduces their overall effectiveness. In the case of Camera 3, the design temperature was not achieved, and the CCD operated at temperatures of $-15{ }^{\circ} \mathrm{C}$ to $0{ }^{\circ} \mathrm{C}$ (depending on the time of year). This was due to high solar thermal input into the camera baffle $(\sim 50 \mathrm{~W})$, insufficient thermal isolation between the baffle and the CCD camera housing, and an inadequate definition of the surrounding spacecraft environment provided prior to the mission (due to technology transfer restrictions). Furthermore, although Cameras 1 and 2 achieved or surpassed the design operating temperature, radiation damage of the CCDs also became an issue for these cameras later in the mission. These issues, especially the "hot pixels" mentioned in Sect. 3.2.1, are minimized by operating the CCDs at a low temperature. A future instrument similar to SMEI would need to address the issue of providing sufficient thermal isolation between the baffle and the CCD housing of any camera looking close to the Sun. Although the incorporation of Peltier coolers was considered early in the design of SMEI, these were not incorporated in the final design primarily because of accommodation difficulties within the very restrictive mechanical constraints of the imaging optics and the $\mathrm{CCD}$, together with the impact on 
the instrument power budget. If a way of accommodating Peltier coolers could be found, they may have enabled a reduction by $\sim 10{ }^{\circ} \mathrm{C}$ to $20^{\circ} \mathrm{C}$ of $\mathrm{CCD}$ temperatures in Cameras 1 and 2 . However, it should be emphasized that their absence was not the primary reason for the elevated temperature of the Camera $3 \mathrm{CCD}$. Even if it had been possible to incorporate a Peltier cooler in Camera 3, it would not have lowered the temperature of this CCD significantly because of its relatively high parasitic thermal load from the high solar thermal load on the baffle of this camera, together with the inherently poor efficiency $(\sim 2-5 \%)$ of Peltier devices. We note also that the "hot pixel" issue described in Sect. 3.2.1 becomes much less important when the CCDs are kept very cold.

\subsubsection{Choice of Detector Technology}

The issues of radiation degradation of CCDs, particularly if their operating temperatures are not sufficiently low, can be avoided by use of Active Pixel Sensors (APS) that can be fabricated using an inherently "rad-hard" technology. For example, these devices are baselined for the focal-plane detectors of all remote-sensing imaging instruments on the Solar Orbiter mission (ESA Solar Orbiter Definition Study Report 2011), because of the potential severe radiation environment. The performance specifications of APS devices are now approaching those of "previous generation" CCDs as used in SMEI and STEREO. Furthermore, their use would offer significant potential savings in terms of mass, size and power, and in the complexity of read-out electronics.

\subsubsection{DHU Radiation Tolerance}

Clearly, a future instrument should be developed at a level where radiation-hardened electronics can be specified, particularly for the CPU of the DHU.

\subsubsection{Response to Soft Electrons in the Polar Regions}

A significant part of the energetic particle effects during passages through the polar regions was believed to be due to low-energy electrons reaching the CCD. This could be mitigated by including a relatively thin optical window somewhere within the optical path. In the case of a design similar to SMEI, a natural location would be between the baffle and optics box.

\subsubsection{Baffle Performance}

SMEI used very deep baffles with a FOV of only about $4^{\circ}$ in their narrow direction to achieve maximum stray-light exclusion. It has since been shown with the STEREO/HIs that a much wider FOV can effectively reduce sunlight further. In a scanning instrument such a design would potentially reduce the amount of sky coverage lost to particle hits and auroral contamination. However, as mentioned above, it remains to be determined whether the Moon would cause too much loss of coverage in a sublunary low-Earth orbit configuration.

\subsubsection{Summary}

To successfully achieve the specifications of Table 1, all of the factors degrading the instrument performance discussed in Sect. 3.2 must be adequately addressed in various ways. These include orbit selection, mission and instrument design, data post-processing, and careful background modeling. We have found that the latter two can alleviate some of the difficulties arising from the former, for example, using post-processing techniques with the STEREO/HI data (DeForest et al. 2011). 


\subsection{Suggestions for Future Heliospheric Imagers}

SMEI has proven not only that CMEs can be observed, tracked, and quantitatively measured during their passage throughout the inner heliosphere, but also that CME information can be extracted from heliospheric imagers that is unavailable from coronagraphs. Along with the large variety of astronomical advances that can also be accomplished, heliospheric imagers have proven to be a crucial resource for both the scientific and the operational communities. With the cessation of data from the SMEI mission, and with the STEREO spacecraft (now in their 7th year of science mission operations) moving considerably behind the Sun relative to the Earth, future heliospheric imaging missions are vital to continue to develop and utilize this unique resource. While heliospheric imagers are currently being developed for the next generation of solar spacecraft observatories (SoloHI for Solar Orbiter and WISPR for Solar Probe +), they are not due for launch until later in the decade (2017 and 2018, respectively), and are very similar instruments to the STEREO/HIs. Other classes of heliospheric imager have been proposed for future missions. We briefly discuss two types proposed by SMEI team members and a third concept.

An important objective for a future heliospheric imager is to take advantage of the lessons learned from SMEI and the HIs (Sects. 6.1 and 6.2) to develop a better quality imager and enable more accurate calibration and scientific data extraction. To this end, Buffington et al. (1998), Jackson et al. (2010a, 2011a) have proposed the All-Sky Imager (ASI). This instrument views almost an entire hemisphere of directions relative to the Sun, from $2^{\circ}$ out as far as $180^{\circ}$. The instrument uses a mirror-lens optical system in order to provide a very large FOV, whilst minimizing scattering of light from bright objects (see Buffington 2000; Buffington et al. 2009c), and would be suitable for deployment far enough away from lowEarth orbit that interference from the Moon would not be a problem.

Recent theoretical work (Howard and Tappin 2009a; Howard and DeForest 2012b; DeForest et al. 2013; Howard et al. 2013) has demonstrated that additional information and kinematic parameter and density calculation precision could be obtained if heliospheric imagers had the capability to observe in polarized as well as unpolarized light. Polarized images contain 3-D information that is not available in unpolarized images, because the Thomson surface (Sect. 4.1.2), which is largely unimportant in unpolarized light (Howard and DeForest 2012a), becomes highly significant in polarized light (DeForest et al. 2013). The concept of a polarizing heliospheric imager has been proposed by Howard (2011).

A special configuration of space weather interest occurred in 2009-2010 when STEREOB was in the vicinity of the L5 Lagrange point which is located $60^{\circ}$ east of the Sun-Earth line. Observations at or near this location permitted the use of STEREO-B as a pathfinder for a space weather monitoring mission that could include heliospheric imaging capabilities (e.g., Webb et al. 2010b). In particular, the speed and size of an Earth-directed CME is perhaps more easily determined from a side view than a head-on view, and that the side view might enable the observation of such a CME at an earlier time than is feasible with the head-on view. In addition, from this location, active regions and coronal holes can be viewed before they arrive on the Earth-facing disk, and their location, size and activity assessed. Finally, the geoeffective space weather resulting from high-speed solar wind streams, such as radiation belt relativistic electron enhancements, can be forecast days in advance from L5. The current understanding of CMEs primarily comes from the SOHO and the STEREO missions, but these missions lacked some key measurements: STEREO did not have a magnetograph and SOHO did not have heliospheric imagers or an in-situ magnetometer. SOHO and other imagers such as SMEI located on the Sun-Earth line are also not ideal for measuring Earthdirected CMEs. The Earth-Affecting Solar Causes Observatory (EASCO, Gopalswamy et al. 
2011 ) is one proposed mission to be located at the Sun-Earth L5 that would overcome these deficiencies. This and other concepts involving L5 heliospheric imagers are mentioned in the NRC 2012 Solar and Space Physics Decadal Survey document. The largest drawbacks of such a concept are cost and telemetry for deploying at and communicating with this distant location.

\subsection{Concluding Remarks}

As discussed throughout this review, SMEI was the first of a new class of solar and space imager. This paper reviews the many accomplishments of SMEI for CME science, astrophysics and space weather. The SMEI team expects this review and the extensive list of references contained within to be useful for authors of future papers investigating various scientific phenomena observed by heliospheric imagers. It is important to note that with the de-activation of SMEI and the HIs now well beyond the optimum geometry for observing Earth-directed CMEs, we are currently in danger of losing the capability to make these unique observations that have been proven to provide invaluable scientific and operational information unavailable from any other dataset. Finally, we hope that those reviewing this work will share our view about the important and urgent need for a next generation heliospheric imager.

Acknowledgements The SMEI instrument was designed and built by a team of scientists and engineers at the University of Birmingham in the UK, the University of California at San Diego (UCSD), together with the Rutherford Appleton Laboratory in the UK, who designed the CCD cameras under a sub-contract to the University of Birmingham. Program management was conducted by the Air Force Research Laboratory (AFRL). Financial support has been provided by the US Air Force, the University of Birmingham, and NASA, and the science projects have been funded by AFRL, NASA and NSF. Data processing pipelines were developed by AFRL, UCSD, and NSO. We thank all of those who helped to make SMEI an unmitigated success: To the Principal Investigators over the years, R. Altrock, S. Keil, R. Radick, J. Johnston, and M. Crown; to the technical team (not listed as authors), P. Anderson, M. Deeley, S. Figueroa, J. Hewitt, D. Hoyland, J. King, R. Fisher, and the late P. Thompson; and to the science teams including those not listed as authors, H. Bruntt, C. Fry, R. Gold, and J. Mozer. Thanks also to RSC, Bach Research, Spectrum Astro. Inc. (the spacecraft contractor), and the Air Force Space Test Program and the US Navy for mission support. Finally, we thank P. Klein, the Coriolis Project Manager and R. Sagalyn for garnering early support for the program. The National Solar Observatory is operated by AURA, Inc., under cooperative agreement with the National Science Foundation. Partial support for NSO is provided by the USAF under a Memorandum of Agreement.

This paper is dedicated in memory of Dale R. Sinclair and Dr. Stephan D. Price, long time consultants to the SMEI program. Their advice and leadership were invaluable to the success of the mission.

Open Access This article is distributed under the terms of the Creative Commons Attribution License which permits any use, distribution, and reproduction in any medium, provided the original author(s) and the source are credited.

\section{References}

M.M. Bisi, B.V. Jackson, P.P. Hick, A. Buffington, D. Odstrĉil, J. M. Clover, J. Geophys. Res. 113, A00A11 (2008). doi:10.1029/2008JA013222

M.M. Bisi, B.V. Jackson, A.R. Breen, G.D. Dorrian, R.A. Fallows, J.M. Clover, P.P. Hick, Sol. Phys. 265, 233-244 (2010)

G.E. Brueckner, R.A. Howard, M.J. Koomen, C.M. Korendyke, D.J. Michels, J.D. Moses, D.G. Socker, K.P. Dere, P.L. Lamy, A. Llebaria, M.V. Bout, R. Schwenn, G.M. Simnett, D.K. Bedford, C.J. Eyles, Sol. Phys. 162, 357-402 (1995)

H. Bruntt, Commun. Asteroseismol. 150, 326 (2007)

H. Bruntt, J. Southworth, G. Torres, A.J. Penny, J.V. Clausen, D.L. Buzasi, Astron. Astrophys. 456, 651-658 (2006)

H. Bruntt, N.R. Evans, D. Stello, A.J. Penny, J.A. Eaton, D.L. Buzasi, D.D. Sasselov, H.L. Preston, E. MillerRicci, Astrophys. J. 683, 433-440 (2008) 
A. Buffington, Appl. Opt. 39, 2683 (2000)

A. Buffington, C.H. Booth, H.S. Hudson, Publ. Astron. Soc. Pac. 103, 685-693 (1991)

A. Buffington, P.P. Hick, B.V. Jackson, C.M. Korendyke, Proc. SPIE 3442, 77-86 (1998)

A. Buffington, B.V. Jackson, P.P. Hick, Proc. SPIE 4853, 490-503 (2003)

A. Buffington, B.V. Jackson, P.P. Hick, Proc. SPIE 5901, 590118 (2005)

A. Buffington, D.L. Band, B.V. Jackson, P.P. Hick, A.C. Smith, Astrophys. J. 637, 880-888 (2006a)

A. Buffington, B.V. Jackson, P. Hick, S.D. Price, EOS Trans. AGU, vol. 87, Fall Meet. Suppl., Abstract SH11A-04 (2006b)

A. Buffington, J.S. Morrill, P.P. Hick, R.A. Howard, B.V. Jackson, D.F. Webb, Proc. SPIE 6689, 66890B (2007)

A. Buffington, M.M. Bisi, J.M. Clover, P.P. Hick, B.V. Jackson, T.A. Kuchar, Astrophys. J. 677, 798-807 (2008)

A. Buffington, M.M. Bisi, J.M. Clover, P.P. Hick, B.V. Jackson, T.A. Kuchar, S.D. Price, Icarus 203, 124-133 (2009a)

A. Buffington, M.M. Bisi, J.M. Clover, P.P. Hick, B. Jackson, EOS Trans. AGU, vol. 90, Fall Meet. Suppl., Abstract SH12A-06 (2009b)

A. Buffington, K.G. Bach, B.W. Bach, E.K. Bach, M.M. Bisi, P.P. Hick, B.V. Jackson, P.D. Klupar, in Proc. SPIE, vol. 7438, ed. by S. Fineschi, J.A. Fennelly (2009c), p. 743800.

A. Buffington, B.V. Jackson, P.P. Hick, J.M. Clover, M.M. Bisi, EOS Trans. AGU, vol. 91, Fall Meet. Suppl., Abstract SH41A-1780 (2010)

E.W. Cliver, A.G. Ling, I.G. Richardson, Astrophys. J. 592, 574-579 (2003)

J.M. Clover, B.V. Jackson, A. Buffington, P.P. Hick, M.M. Bisi, Astrophys. J. 713, 394-397 (2010)

C.E. DeForest, T.A. Howard, S.J. Tappin, Astrophys. J. 738, 103-112 (2011)

C.E. DeForest, T.A. Howard, S.J. Tappin, Astrophys. J. 765, 44 (2013)

M. Dryer, Z.K. Smith, G.H. Endrud, J.H. Wolfe, Cosm. Electrodyn. 3, 184-207 (1972)

T. Dunn, B.V. Jackson, P.P. Hick, A. Buffington, X.P. Zhao, Sol. Phys. 227, 339-353 (2005)

E. Echer, M.V. Alves, W.D. Gonzalez, J. Atmos. Sol.-Terr. Phys. 67, 839-852 (2005)

European Space Agency, Solar Orbiter Definition Study Report, ESA/SRE [2011]14 (2011)

C.J. Eyles, G.M. Simnett, M.P. Cooke, B.V. Jackson, A. Buffington, P.P. Hick, N.R. Waltham, J.M. King, P.A. Anderson, P.E. Holladay, Sol. Phys. 217, 319-347 (2003)

C.J. Eyles, R.A. Harrison, C.J. Davis, N.R. Waltham, B.M. Shaughnessy, H.C.A. Mapson-Menard, D. Bewsher, S.R. Crothers, J.A. Davies, G.M. Simnett, R.A. Howard, J.D. Moses, J.S. Newmark, D.G. Socker, J.-P. Halain, J.-M. Defise, E. Mazy, P. Rochus, Sol. Phys. 254, 387-445 (2009)

S.E. Forbush, J. Geophys. Res. 59, 525-542 (1954)

C.D. Fry, W. Sun, C.S. Deehr, M. Dryer, Z. Smith, S.-I. Akasofu, M. Tokumaru, M. Kojima, J. Geophys. Res. 106, 20985-21002 (2001)

P.W. Gaiser, IEEE Trans. Geosci. Remote Sens. 42, 2347-2361 (2004)

N. Gopalswamy, S. Yashiro, G. Michalek, H. Xie, P. Mäkelä, A. Vourlidas, R.A. Howard, Sun Geosph. 5, 7-16 (2010)

N. Gopalswamy, J.M. Davila, F. Auchère, J. Schou, C. Korendyke, A. Shih, J.C. Johnston, R.J. MacDowall, M. Maksimovic, E. Sittler, A. Szabo, R. Wesenberg, S. Vennerstrom, B. Heber, in Solar Physics and Space Weather Instrumentation IV, ed. by S. Fineschi, J. Fennelly. Proc. SPIE, vol. 8148 (2011), p. 81480Z. doi:10.1117/12.901538

P.P. Hick, B.V. Jackson, Space Sci. Rev. 97, 35-38 (2001)

P.P. Hick, B.V. Jackson, in Proc. SPIE, vol. 5171, ed. by S. Fineschi, M.A. Gummin (2004), pp. 287-297

P.P. Hick, A. Buffington, B.V. Jackson, in Proc. SPIE, vol. 5901, ed. by S. Fineschi, R.A. Viereck (2005), p. 59011B

P.P. Hick, A. Buffington, B.V. Jackson, in Proc. SPIE, vol. 6689, ed. by S. Fineschi, R.A. Viereck (2007), p. $66890 \mathrm{C}$

R. Hounsell, M.F. Bode, P.P. Hick, A. Buffington, B.V. Jackson, J.M. Clover, A.W. Shafter, M.J. Darnley, N.R. Mawson, I.A. Steele, A. Evans, S.P.S. Eyres, T.J. O’Brien, Astrophys. J. 724, 480-486 (2010)

D. Hovestadt, M. Hilchenbach, A. Bürgi, B. Klecker, P. Laeverenz, M. Scholer, H. Grünwaldt, W.I. Axford, S. Livi, E. Marsch, B. Wilken, H.P. Winterhoff, F.M. Ipavich, P. Bedini, M.A. Coplan, A.B. Galvin, G. Gloeckler, P. Bochsler, H. Balsiger, J. Fischer, J. Geiss, R. Kallenbach, P. Wurz, K.-U. Reiche, F. Gliem, D.L. Judge, H.S. Ogawa, K.C. Hsieh, E. Möbius, M.A. Lee, G.G. Managadze, M.I. Verigin, M. Neugebauer, Sol. Phys. 162, 441-481 (1995)

T.A. Howard, J. Atmos. Sol.-Terr. Phys. 73, 1242-1253 (2011)

T.A. Howard, C.E. DeForest, Astrophys. J. 746, 64-75 (2012a)

T.A. Howard, C.E. DeForest, Astrophys. J. 752, 130-142 (2012b)

T.A. Howard, G.M. Simnett, J. Geophys. Res. 113, A08102 (2008). doi:10.1029/2007JA012920

T.A. Howard, S.J. Tappin, Astron. Astrophys. 440, 373-383 (2005a) 
T.A. Howard, S.J. Tappin, Geophys. Res. Lett. 32, L14106 (2005b). doi:10.1029/2005GL023056

T.A. Howard, S.J. Tappin, EOS Trans. AGU, vol. 89, Fall Meet. Suppl., Abstract SH13B-1531 (2008)

T.A. Howard, S.J. Tappin, Space Sci. Rev. 147, 31-54 (2009a)

T.A. Howard, S.J. Tappin, Space Sci. Rev. 147, 89-110 (2009b)

T.A. Howard, S.J. Tappin, Space Weather 7, S07004 (2010). doi:10.1029/2009SW000531

R.A. Howard, N.R. Sheeley Jr., M.J. Koomen, Astrophys. J. 263, L101-L104 (1982)

R.A. Howard, N.R. Sheeley Jr., M.J. Koomen, D.J. Michels, J. Geophys. Res. 90, 8173-8191 (1985)

T.A. Howard, G.M. Simnett, I.M. Robinson, J. Tappin, EOS Trans. AGU, vol. 85, Fall Meet. Suppl., Abstract SH11A-04 (2004)

T.A. Howard, D.F. Webb, S.J. Tappin, D.R. Mizuno, J.C. Johnston, J. Geophys. Res. 111, A04105 (2006). doi:10.1029/2005JA011349

T.A. Howard, C.D. Fry, J.C. Johnston, D.F. Webb, Astrophys. J. 667, 610-625 (2007)

T.A. Howard, C.E. DeForest, A.A. Reinard, Astrophys. J. 754, 102-111 (2012)

T.A. Howard, S.J. Tappin, D. Odstrcil, C.E. DeForest, Astrophys. J. 765, 45 (2013)

B.V. Jackson, Sol. Phys. 95, 363-370 (1985a)

B.V. Jackson, Sol. Phys. 100, 563-574 (1985b)

B.V. Jackson, P.P. Hick, Solar and Space Weather Radiophysics (Springer, New York, 2004), p. 355

B.V. Jackson, C. Leinert, J. Geophys. Res. 90, 10759-10764 (1985)

B.V. Jackson, H.S. Hudson, J.D. Nichols, R.E. Gold, in Solar System Plasma Physics, ed. by J.H. Waite Jr., J.L. Burch, R.L. Moore. Geophysical Monograph, vol. 54 (1989), pp. 291-297

B.V. Jackson, P.L. Hick, M. Kojima, A. Yokobe, Adv. Space Res. 20, 23 (1997)

B.V. Jackson, P.L. Hick, M. Kojima, A. Yokobe, J. Geophys. Res. 103, 12049-12068 (1998)

B.V. Jackson, A. Buffington, P.P. Hick, R.C. Altrock, S. Figueroa, P.E. Holladay, J.C. Johnston, S.W. Kahler, J.B. Mozer, S. Price, R.R. Radick, R. Sagalyn, D. Sinclair, G.M. Simnett, C.J. Eyles, M.P. Cooke, S.J. Tappin, T. Kuchar, D.R. Mizuno, D.F. Webb, P.A. Anderson, S.L. Keil, R.E. Gold, N.R. Waltham, Sol. Phys. 225, 177-207 (2004)

B.V. Jackson, A. Buffington, P.P. Hick, X. Wang, D. Webb, J. Geophys. Res. 111, A04S91 (2006). doi:10.1029/2004JA010942

B.V. Jackson, J.A. Boyer, P.P. Hick, A. Buffington, M.M. Bisi, D.H. Crider, Sol. Phys. 241, 385-396 (2007a)

B.V. Jackson, P.P. Hick, A. Buffington, M.M. Bisi, M. Kojima, M. Tokumaru, Astron. Astrophys. Trans. 26, 477-487 (2007b)

B.V. Jackson, A. Buffington, P.P. Hick, M.M. Bisi, E.A. Jenson, Proc. SPIE 6689, 66890G (2007c)

B.V. Jackson, M.M. Bisi, P.P. Hick, A. Buffington, J.M. Clover, W. Sun, J. Geophys. Res. 113, A00A15 (2008). doi:10.1029/2008JA013224

B.V. Jackson, A. Buffington, P.P. Hick, M.M. Bisi, J.M. Clover, in Proc. 3rd Solar Orbiter Workshop (2009a)

B.V. Jackson, M.M. Bisi, P.P. Hick, A. Buffington, J.M. Clover, Proc. AGU Fall Meeting, EOS Trans. 90, Abstract SH11A-1499 (2009b)

B.V. Jackson, A. Buffington, P.P. Hick, M.M. Bisi, J.M. Clover, Sol. Phys. 265, 257-275 (2010a)

B.V. Jackson, A. Buffington, P.P. Hick, J.M. Clover, M.M. Bisi, D.F. Webb, Astrophys. J. 724, 829-834 (2010b)

B.V. Jackson, P.P. Hick, A. Buffington, M.M. Bisi, J.M. Clover, M. Tokumaru, K. Fujiki, in Proc. Solar Wind 12 Workshop. AIP, vol. 1216 (2010c), pp. 659-662

B.V. Jackson, A. Buffington, M.M. Bisi, J. Clover, P.P. Hick, in Proc. 2nd Remote Sensing of the Inner Heliosphere Workshop (2011a), p. 19

B.V. Jackson, P.P. Hick, A. Buffington, M.M. Bisi, J.M. Clover, M. Tokumaru, M. Kojima, K. Fujiki, J. Atmos. Sol.-Terr. Phys. 73, 1214-1227 (2011b)

B.V. Jackson, M.S. Hamilton, P.P. Hick, A. Buffington, M.M. Bisi, J.M. Clover, M. Tokumaru, K. Fujiki, J. Atmos. Sol.-Terr. Phys. 73, 1317-1329 (2011c)

B.V. Jackson, P.P. Hick, A. Buffington, J.M. Clover, M. Tokumaru, Adv. Geosci. 30, 93-115 (2012)

E.A. Jensen, P.P. Hick, M.M. Bisi, B.V. Jackson, J. Clover, T. Mulligan, Sol. Phys. 265, 31-48 (2010)

J.C. Johnston, J.B. Mozer, R.R. Radick, P.E. Holladay, T.A. Kuchar, D.R. Mizuno, D.F. Webb, EOS Trans. AGU, vol. 85, Fall Meet. Suppl., Abstract SH11A-08 (2004)

S.W. Kahler, G.M. Simnett, in Proc. 29th Int. Cosmic Ray Conf, vol. 2, Pune, India (2005), pp. 267-270

S.W. Kahler, D.F. Webb, J. Geophys. Res. 112, A09103 (2007). doi:10.1029/2007JA012358

S.W. Kahler, D.F. Webb, in CP1216. Proc. 12th Int. Solar Wind Conf., ed. by M. Maksimovic, K. Issautier, N. Meyer-Vernet, M. Moncuquet, F. Pentellini (2010), p. 408

M.L. Kaiser, T.A. Kucera, J.M. Davila, O.C. St. Cyr, M. Guhathakurta, E. Christian, Space Sci. Rev. 136, 5-16 (2008)

M. Kojima, M. Tokumaru, H. Watanabe, A. Yokobe, K. Asai, B.V. Jackson, P.L. Hick, J. Geophys. Res. 103, 1981-1990 (1998) 
K.E. Kraemer, S.D. Price, M. Kendra, J. Gibson, T.A. Kuchar, D.R. Mizuno, M. Noah, R.A. Dressler, A. Makuch, AFRL-RV-PS-TR-2011-0132, 1-167 (2011)

T.A. Kuchar, D. Mizuno, C.N. Arge, D.F. Webb, S.W. Kahler, J.C. Johnston, EOS Trans. AGU, vol. 85, Fall Meet. Suppl., Abstract SH21A-0396 (2004)

T.A. Kuchar, A. Buffington, T. Howard, C.N. Arge, D.F. Webb, B.V. Jackson, P.P. Hick, EOS Trans. AGU, vol. 87, Fall Meet. Suppl., Abstract SH32A-08 (2006)

T.A. Kuchar, A. Buffington, C.N. Arge, P.P. Hick, T.A. Howard, B.V. Jackson, J.C. Johnston, D.R. Mizuno, S.J. Tappin, D.F. Webb, J. Geophys. Res. 113, A04101 (2008). doi:10.1029/2007JA012603

J.B. Leighly, Geogr. Rev. 45, 246 (1955)

C. Leinert, H. Link, E. Pitz, N. Salm, D. Kluppelberg, Raumfahrtforschung 19, 264-267 (1975)

J. Li, D. Jewitt, J.M. Clover, B.V. Jackson, Astrophys. J. 728, 31-39 (2011)

N. Lugaz, A. Vourlidas, I.I. Roussev, Ann. Geophys. 27, 3479-3488 (2009)

M.A. Lyons, G.M. Simnett, Sol. Phys. 200, 202-211 (2001)

K. Marubashi, Adv. Space Res. 26, 55-66 (2000)

D.R. Mizuno, A. Buffington, M.P. Cooke, C.J. Eyles, P.P. Hick, P.E. Holladay, B.V. Jackson, J.C. Johnston, T.A. Kuchar, J.B. Mozer, S.D. Price, R.R. Radick, G.M. Simnett, D. Sinclair, S.J. Tappin, D.F. Webb, J. Geophys. Res. 110, A07230 (2005). doi:10.1029/2004JA010689

D.R. Mizuno, S.D. Price, K.E. Kraemer, T.A. Kuchar, J.C. Johnston, Adv. Space Res. 49, 162-176 (2011)

D. Odstrĉil, P. Riley, X.P. Zhao, J. Geophys. Res. 109, A02116 (2004). doi:10.1029/2003JA010135

K.W. Ogilvie, L.F. Burlaga, Sol. Phys. 8, 422-434 (1969)

A.J. Penny, EOS Trans. AGU, vol. 87, Fall Meet. Suppl., Abstract SH32A-07 (2006)

A.J. Penny, B.V. Jackson, A. Buffington, P.P. Hick, S.W. Kahler, S. Price, J.C. Johnston, P. Holladay, D. Sinclair, R.R. Radick, J.C. Mozer, P. Anderson, G.M. Simnett, C.J. Eyles, M.P. Cooke, J. Tappin, N.R. Waltham, T. Kuchar, D. Mizuno, D.F. Webb, EOS Trans. AGU, vol. 84, Fall Meet. Suppl., Abstract SH41C-08 (2003)

I.G. Richardson, D.F. Webb, J. Zhang, D.B. Berdichevsky, D.A. Biesecker, J.C. Kasper, R. Kataoka, J.T. Steinberg, B.J. Thompson, C.-C. Wu, A.N. Zhukov, J. Geophys. Res. 111, A07S09 (2006). doi:10.1029/2005JA011476

I.G. Richardson, D.F. Webb, J. Zhang, D.B. Berdichevsky, D.A. Biesecker, J.C. Kasper, R. Kataoka, J.T. Steinberg, B.J. Thompson, C.-C. Wu, A.N. Zhukov, J. Geophys. Res. 112, A12105 (2007). doi:10.1029/2007JA012332

I. Richter, C. Leinert, B. Planck, Astron. Astrophys. 110, 115-120 (1982)

E. Robbrecht, D. Berghmans, Astron. Astrophys. 425, 1097-1106 (2004)

E. Robbrecht, D. Berghmans, R.A.M. van der Linden, Astrophys. J. 691, 1222-1234 (2009)

A.P. Rouillard, J.A. Davies, R.J. Forsyth, A. Rees, C.J. Davis, R.A. Harrison, M. Lockwood, D. Bewsher, S.R. Crothers, C.J. Eyles, M. Hapgood, C.H. Perry, Geophys. Res. Lett. 35, L10110 (2008). doi:10.1029/2008GL033767

N.R. Sheeley Jr., J.H. Walters, Y.-M. Wang, R.A. Howard, J. Geophys. Res. 104, 24739-24768 (1999)

N.R. Sheeley Jr., A.D. Herbst, C.A. Palatchi, Y.-M. Wang, R.A. Howard, J.D. Moses, A. Vourlidas, J.S. Newmark, D.G. Socker, S.P. Plunkett, C.M. Korendyke, L.F. Burlaga, J.M. Davila, W.T. Thompson, O.C. St. Cyr, R.A. Harrison, C.J. Davis, C.J. Eyles, J.P. Halain, D. Wang, N.B. Rich, K. Battams, E. Esfandiari, G. Stenborg, Astrophys. J. 674, L109-L112 (2008)

G.M. Simnett, in Proc. Solar Wind 11/SOHO 16, ed. by B. Fleck, T.H. Zurbuchen, H. Lacoste. ESA SP, vol. 592 (ESTEC, Noordwijk, 2005), p. 767

G.M. Simnett, T.A. Howard, EOS Trans. AGU, vol. 87, Fall Meet. Suppl., Abstract SH32A-05 (2006)

G.M. Simnett, S.W. Kahler, EOS Trans. AGU, vol. 85, Fall Meet. Suppl., Abstract SH21A-0392 (2004)

G.M. Simnett, S.W. Kahler, in Proc. 29th Int. Cosmic Ray Conf, vol. 1, Pune, India (2005), pp. 165-168

E.J. Smith, J.H. Wolfe, Geophys. Res. Lett. 3, 137-140 (1976)

S.A. Spreckley, I.R. Stevens, Mon. Not. R. Astron. Soc. 388, 1239-1244 (2008)

E.C. Stone, A.M. Frandsen, R.A. Mewaldt, E.R. Christian, D.E. Margolies, J.F. Ormes, F. Snow, Space Sci. Rev. 86, 1-22 (1998)

C. Størmer, in The Polar Aurora (Clarendon, Oxford, 1955)

W. Sun, C.S. Deehr, C.D. Fry, M. Dryer, Z. Smith, S.-I. Akasofu, Space Weather 6, L02802 (2008). doi:10.1029/2006SW000298

S.J. Tappin, Sol. Phys. 233, 233-248 (2006)

S.J. Tappin, T.A. Howard, Space Sci. Rev. 147, 55-87 (2009a)

S.J. Tappin, T.A. Howard, Astrophys. J. 702, 862-870 (2009b)

S.J. Tappin, A. Buffington, M.P. Cooke, C.J. Eyles, P.P. Hick, P.E. Holladay, B.V. Jackson, J.C. Johnston, T. Kuchar, D. Mizuno, J.B. Mozer, S. Price, R.R. Radick, G.M. Simnett, D. Sinclair, N.R. Waltham, D.F. Webb, Geophys. Res. Lett. 31, L02802 (2004). doi:10.1029/2003GL018766 
S.J. Tappin, T.A. Howard, M.M. Hampson, R.N. Thompson, C.E. Burns, J. Geophys. Res. 117, A05103 (2012). doi:1029/2011JA017439

N.J. Tarrant, W.J. Chaplin, Y. Elsworth, S.A. Spreckley, I.R. Stevens, Astron. Astrophys. 483, L43-L46 (2008a)

N.J. Tarrant, W.J. Chaplin, Y. Elsworth, S.A. Spreckley, I.R. Stevens, Astron. Astrophys. 492, 167-169 (2008b)

N.J. Tarrant, W.J. Chaplin, Y.P. Elsworth, S.A. Spreckley, I.R. Stevens, Commun. Asteroseismol. 157, 92-97 (2008c)

H.E. Taylor, Sol. Phys. 6, 320-334 (1969)

M. Tokumaru, M. Kojima, K. Fujiki, M. Yamashita, B.V. Jackson, J. Geophys. Res. 112, A05106 (2007). doi:10.1029/2006JA012043

A. Vourlidas, R.A. Howard, Astrophys. J. 642, 1216-1221 (2006)

A. Vourlidas, C.J. Davis, C.J. Eyles, S.R. Crothers, R.A. Harrison, R.A. Howard, J.D. Moses, D.G. Socker, Astrophys. J. 668, L79-L82 (2007)

D.F. Webb, J.C. Johnson, R.R. Radick, the SMEI Team, EOS Trans. AGU 83(33), 38-39 (2002)

D.F. Webb, D.R. Mizuno, A. Buffington, M.P. Cooke, C.J. Eyles, C.D. Fry, L.C. Gentile, P.P. Hick, P.E. Holladay, T.A. Howard, J.G. Hewitt, B.V. Jackson, J.C. Johnston, T.A. Kuchar, J.B. Mozer, S. Price, R.R. Radick, G.M. Simnett, S.J. Tappin, J. Geophys. Res. 111, A12101 (2006). doi:10.1029/2006JA011655

D.F. Webb, T.A. Howard, C.D. Fry, T.A. Kuchar, D. Odstrĉil, B.V. Jackson, M.M. Bisi, R.A. Harrison, J.S. Morrill, R.A. Howard, J.C. Johnston, Sol. Phys. 256, 239-267 (2009a)

D.F. Webb, T.A. Howard, C.D. Fry, T.A. Kuchar, D.R. Mizuno, J.C. Johnston, B.V. Jackson, Space Weather 7, S05002 (2009b). doi:10.1029/2008SW000409

D.F. Webb, J.C. Johnston, T.A. Kuchar, J. Tappin, T.A. Howard, EOS Trans. AGU, vol. 91, Fall Meet. Suppl., Abstract SH54D-05 (2010a)

D.F. Webb, D.A. Biesecker, N. Gopalswamy, O.C. St. Cyr, J.M. Davila, C.J. Eyles, B.J. Thompson, K.D.C. Simunac, J.C. Johnston, Space Res. Today 178, 10-16 (2010b)

D.F. Webb, C. Möstl, B.V. Jackson, M.M. Bisi, T.A. Howard, T. Mulligan, E.A. Jensen, L.K. Jian, J.A. Davies, C.A. de Koning, Y. Liu, M. Temmer, J.M. Clover, C.J. Farrugia, R.A. Harrison, N. Nitta, D. Odstrĉil, S.J. Tappin, H.-S. Yu, Sol. Phys. 285, 317 (2013)

G. Wibberenz, I.G. Richardson, H.V. Cane, J. Geophys. Res. 107, 1353 (2002). doi:10.1029/2002JA009461

R.M. Wilson, J. Geophys. Res. 95, 215-219 (1990)

M. Xiong, A.R. Breen, M.M. Bisi, M.J. Owens, R.A. Fallows, G.D. Dorrian, J.A. Davies, P. Thomasson, J. Atmos. Sol.-Terr. Phys. 73, 1270-1280 (2011)

M. Xiong, J.A. Davies, M.M. Bisi, M.J. Owens, R.A. Fallows, G.D. Dorrian, Sol. Phys. (2012, in press)

Y. Yu, P.P. Hick, B.V. Jackson, Proc. SPIE 5901, 335-339 (2005)

J. Zhang, I.G. Richardson, D.F. Webb, N. Gopalswamy, E. Huttunen, J.C. Kasper, N.V. Nitta, W. Poomvises, B.J. Thompson, C.-C. Wu, S. Yashiro, A.N. Zhukov, J. Geophys. Res. 112, A10102 (2007a). doi:10.1029/2007JA012321

J. Zhang, I.G. Richardson, D.F. Webb, N. Gopalswamy, E. Huttunen, J.C. Kasper, N.V. Nitta, W. Poomvises, B.J. Thompson, C.-C. Wu, S. Yashiro, A.N. Zhukov, J. Geophys. Res. 112, A12103 (2007b). doi:10.1029/2007JA012891 